PAPER

\title{
Attosecond light science and its application for probing quantum materials
}

\section{Recent citations}

- Ultrafast extreme ultraviolet photoemission electron microscope

Wei Zheng et al

To cite this article: Xun Shi et al 2020 J. Phys. B: At. Mol. Opt. Phys. 53184008

View the article online for updates and enhancements.

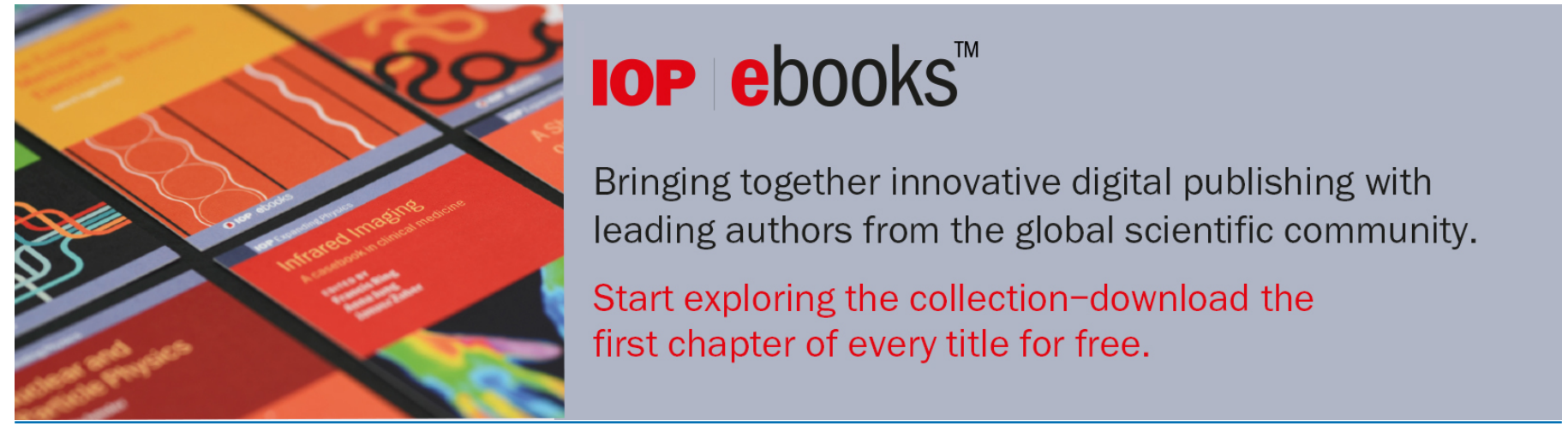

This content was downloaded from IP address 212.128.135.32 on 14/04/2021 at 15:30 


\title{
Attosecond light science and its application for probing quantum materials
}

\author{
Xun Shi ${ }^{1,4}{ }^{\oplus}$, Chen-Ting Liao ${ }^{1} \oplus$, Zhensheng Tao $^{2}$, \\ Emma Cating-Subramanian ${ }^{1}\left({ }^{1}\right.$, Margaret M Murnane ${ }^{1,4}$, \\ Carlos Hernández-García ${ }^{3}$ and Henry C Kapteyn ${ }^{1}$ \\ ${ }^{1}$ Department of Physics and JILA, University of Colorado and NIST, Boulder, CO 80309, United States \\ of America \\ 2 State Key Laboratory of Surface Physics and Department of Physics, Fudan University, Shanghai \\ 200438, People's Republic of China \\ 3 Grupo de Investigación en Aplicaciones del Láser y Fotónica, Departamento de Física Aplicada, \\ University of Salamanca, Salamanca E-37008, Spain \\ E-mail: Xun.Shi@colorado.edu and Margaret.Murnane@colorado.edu
}

Received 1 April 2020, revised 12 May 2020

Accepted for publication 6 July 2020

Published 6 August 2020

\begin{abstract}
In this paper, we review the development and application of coherent short wavelength light sources implemented using the high harmonic generation (HHG) process. The physics underlying HHG brought quantum physics into the domain of attosecond time-scales for the first time. The observation and manipulation of electron dynamics on such short time-scales — a capability not conceived — of just a few decades ago-is becoming both more-and-more sophisticated and useful as a route to achieve exquisite control over short wavelength light. New experimental techniques are enabling HHG light sources to provide new insights into fundamental quantum interactions in materials, making it possible for the first time to capture the fastest charge, spin, photon and phonon interactions and to achieve diffraction-limited imaging at short wavelengths.
\end{abstract}

Keywords: high harmonic generation, attosecond, ultrafast dynamics, spin and orbital angular momentum, angle-resolved photoemission spectroscopy, coherent diffraction imaging

(Some figures may appear in colour only in the online journal)

\section{Introduction and evolution}

The fastest intrinsic interactions and dynamics in atomic, molecular, and solid state systems occur on attosecond timescales on up. The excitation energies corresponding to these processes are governed by the uncertainty principle: $\Delta E \Delta t \geqslant \hbar / 2$, or approximately time scale $(\mathrm{fs}) \sim$ $1 /$ energy scale $(\mathrm{eV})$. Thus, most lower-energy intrinsic dynamics corresponding to the interaction of charges with molecular vibrations and phonons occur on femtosecond and longer timescales. However, higher-energy excitations $(>\mathrm{eV})$ that support attosecond timescale processes are increasingly relevant to a broad range of science and technology.

\footnotetext{
${ }^{4}$ Authors to whom any correspondence should be addressed.
}

The first era in attosecond science evolved from explorations of how atoms and molecules ionize in intense laser fields. This led to the discovery of high-harmonic generation (HHG) - essentially the coherent version of the Röntgen x-ray tube-which still remains an exciting research area after $\sim 3$ decades [1-4]. In the HHG process, attosecond dynamics are induced or driven by using a femtosecond laser pulse to ionize matter. Laser light - a coherent electromagnetic wave with a period on the order of a few femtoseconds-can rapidly push electrons around in matter. At low intensities, this response is linear and harmonic, where electrons are driven sinusoidally by the laser field. However, in the strong field regime, the electron motion is extreme: on each half-cycle of the laser, the electron tunnel ionizes, moves away from the ion, before recolliding to emit excess 
kinetic energy as an HHG photon. In a quantum picture, the strong field ionization process creates a laser-driven nanoscale quantum antenna: the laser field spatially and temporally modulates the atomic electron wavefunction on $\AA$ spatial and attosecond temporal scales. These induced oscillations directly result in the emission of high- energy high harmonics, with a large spectral region (plateau) where harmonic spectral peaks have comparable amplitude. The emission is coherent, and the behavior is very different from the rapidly diminishing yield expected from perturbative nonlinear optics. Even very early-on, quantum simulations showed that this could be explained by coherent attosecond time-scale recollision dynamics [5].

The second era of attosecond science came with the development and application of intense ultrashort-pulse ( $\sim$ sub-10 cycles) lasers [6-8] to HHG [9]. By using a light field that varied on a cycle-by-cycle basis, it became possible to directly see the manifestations of these attosecond dynamics in the spectrum of the emission, in a way that could be directly manipulated [10, 11] and compared with theory [12], settling questions of the origin of HHG. Coherent control of HHG compellingly demonstrated that these attosecond quantum dynamics were not simply of academic interest, but could be usefully manipulated [13]. Phase-matching of the process [14] —a mechanism only practical with few-cycle light pulses - made it possible to dramatically enhanced the brightness, coherence, photon energy range and control of HHG. By understanding and mastering phase matching - where the HHG emission from many atoms coherently combines-has allowed us to fully-optimize the conversion of laser light to shorter wavelength photons, providing a very-useful flux of extreme ultraviolet (EUV) and soft x-ray (SXR) light [14-16]. Indeed, femtosecond lasers, when combined with the HHG process, gave us the first 'tabletop x-ray laser', producing laser-like beams spanning the ultraviolet (UV) and EUV region. The number of bursts in the attosecond pulse train created by HHG could be shortened to a single one [17, 18] using even-shorter $<5$-cycle driving pulses [19]. Aided further by the time-domain dynamics of the phase matching $[20,21]$.

The past decade has shown an exquisite ability to harness and control the attosecond dynamics of the HHG process, allowing for unprecedented control over SXR light. The region of bright HHG has now been extended from the EUV to the $\mathrm{keV}$ photon energy range by using mid-infrared (mid-IR) driving lasers to extend the phase matching range [21-23]. More recently, it is now possible to exquisitely control the HHG polarization [24-30] and orbital angular momentum (OAM) structure $[31,32]$ by controlling the driving laser beams. At this point, the most-complex coherent electromagnetic fields ever created reflect our ability to control the attosecond timescale dynamics of radiating electron wave functions [27]. Moreover, completely new states of light can be created that present a convincing case that HHG represents an unprecedented degree of control over electromagnetic radiation-far more control than we have ever achieved with conventional laser technology —on sub- $\AA$ spatial scales and sub-attosecond temporal scales. Recent developments in these exciting capabilities are further elaborated-on in section 2 .
However, HHG is fundamentally an extrinsic attosecond process i.e. the attosecond dynamics are driven by intense femtosecond lasers. Another longstanding goal of attosecond science has been to observe intrinsic attosecond timescale dynamics i.e. to directly observe the fastest charge and spin dynamics in matter with energy scales that naturally support the fastest attosecond timescales. Fortunately, this has recently been achieved experimentally and marks the third era of attosecond science. Section 3 discusses the relevance of attosecond timescales - and their direct measurement- to capture the fastest charge dynamics in materials systems. We describe our recent studies demonstrating that band structure in materials far above the Fermi level can strongly influence the lifetime of electrons excited into these bands [33]. Furthermore, we discuss how electron-electron scattering and screening can be distinguished through attosecond metrology techniques [34]. Interestingly, these measurements highlighted the counterintuitive finding that attosecond pulse trains are best for measuring intrinsic sub-femtosecond dynamics, while isolated attosecond pulses are ideal for measuring 1-5 fs dynamics.

In section 4 we review the broader application of HHG sources to uncover exciting new physics. The magnetic and phase properties of materials can be directly manipulated by light - on attosecond, femtosecond, and picosecond timescales, depending on the nature of the coupled charge/spin/phonon/photon physics [35-37]. High harmonic beams are ideal as the illumination source for time- and angle-resolved photoemission spectroscopy (trARPES) and element-specific magneto-optic Kerr effect (EUV-MOKE) spectroscopy. Using a new technique we call ultrafast electron calorimetry $[38,39]$, we made a number of new observations of how ultrafast lasers can be used to delicately manipulate materials properties, that uncover a fundamental new understanding of phase transitions and interactions in strongly-coupled quantum materials. The new behavior observed appears to be universal in many materials, and can guide the development of a more complete theoretical understanding of the interactions between charges, spins, and phonons in materials. The forte of femtosecond timeresolved pump-probe techniques is the large separation in timescales between electron-electron, electron-photon, and electron-spin interactions, compared with electron-phonon and phonon dynamics in materials. Thus, Frontier areas such as ultrafast photoemission and magnetic spectroscopies are increasingly being driven by the use of HHG light sources.

Finally, in section 5, we speculate about the next era of attosecond science in terms of broader impact and applications of $\mathrm{HHG}$ - with potential for impact beyond basic science and for a societal return on investment. The ability to generate fully coherent light at short wavelengths makes it possible to implement a tabletop-scale microscope with near-perfect resolution and unique capabilities - a new record optical resolution in this category [40-42]. This ability has immediate implications for nanotechnology, nanoelectronics, and in-future for biomedical applications, and its widespread application outside its area of origin will mark the fourth era of attosecond science (figure 1). 


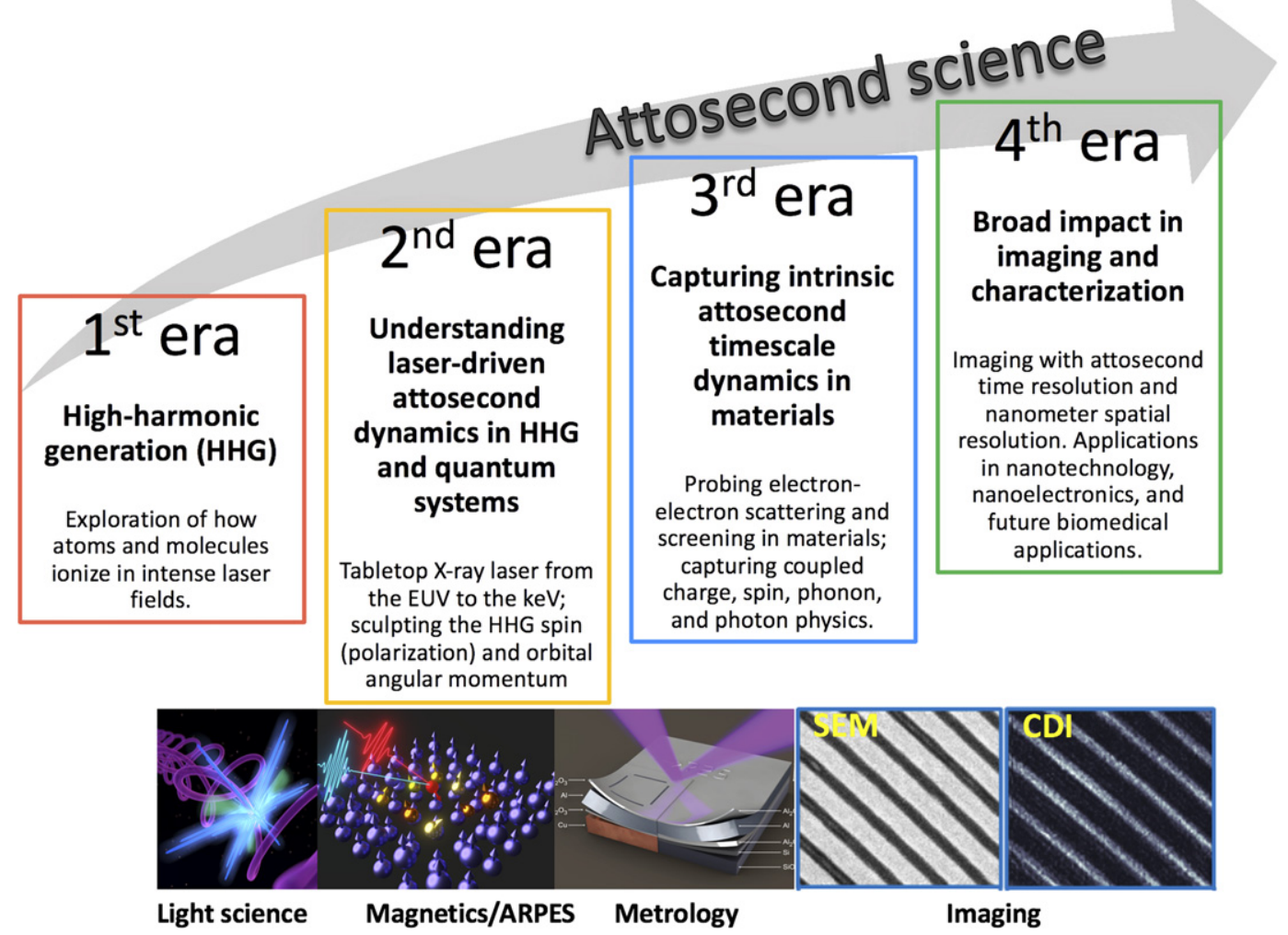

Figure 1. The progression of attosecond science and its applications for probing quantum systems.

\section{New developments in HHG sources: full control over the waveform, spectrum, polarization and OAM structure in the EUV and SXR regions}

\subsection{Introduction}

In recent decades, our ability to structure the spectral and temporal waveform, polarization, phase, intensity, and angular momentum of light has revolutionized imaging and sensing across all wavelength ranges. HHG is a unique EUV/SXR light source because it enables ultra-precise control over light by manipulating the wavefunction of a radiating electron. This makes it possible to control the spectral and temporal waveform and spectrum by controlling the quantum or intrinsic phase in multiple ways, or harnessing phase matching to sculpt the temporal emission window.

One of the most straightforward ways to modulate the temporal and spectral waveform of HHG is to change the driving laser wavelength. When HHG is driven by Ti:sapphire lasers at wavelengths in the near-IR and visible region, bright EUV harmonics (up to the phase matching limit of $\sim 150 \mathrm{eV}$ ) consisting of attosecond pulse trains can be generated. When optimally phase matched, a photon flux in the $>3 \times 10^{12}$ photons/s/1\% bandwidth can be generated using only a $\sim 4 \mathrm{~W}$ driving laser (note that this corresponds to $\sim 19 \mu \mathrm{W}$ in a single harmonic at the source, around $40 \mathrm{eV}$ photon energy, when driven by a $4 \mathrm{~W}$ laser). Then, as the wavelength of the laser light is increased, so also is the phase matching limit, allowing practical fully-coherent HHG sources to be implemented throughout the SXR regime, to $>\mathrm{keV}$ photon energies [21-23, 43-45] - and in the future, possibly into the hard x-ray regime [43, 46]. Moreover, the phase matching window reduces from multiple laser cycles of a near-IR driving laser, to a single half-cycle in the case of a $\sim 2-4 \mu \mathrm{m}$ mid-IR driving laser [47, 48]. This naturally creates a coherent SXR supercontinuum, spanning 12 octaves. The extent to which HHG can be controlled-where the X-ray wavefronts are synchronized at the sub- $\AA$, sub-attosecond level or no beam could emerge - is quite remarkable, enabled by the fundamental quantum physics and the extreme nonlinear nature of this process.

In recent exciting advances we discuss below, selection rules make it possible to also control the polarization, divergence, and orbital angular momentum structure of HHG - simply by modulating the wavelength, geometry, or structure of the femtosecond driving laser(s) [22, 25, 31, 49-51]. For example, when HHG is driven by laser beams with different orbital angular momentum (OAM) values, the selection rules control the OAM of the different harmonics that are generated, and different OAM values have different divergences. These open up new applications of HHG for probing and imaging dynamics in chiral systems.

\subsection{Sculpting the angular momentum of high-order harmonic beams}

HHG can generate ultrashort pulses not only with unique temporal and spectral properties, but also with custom and unique 

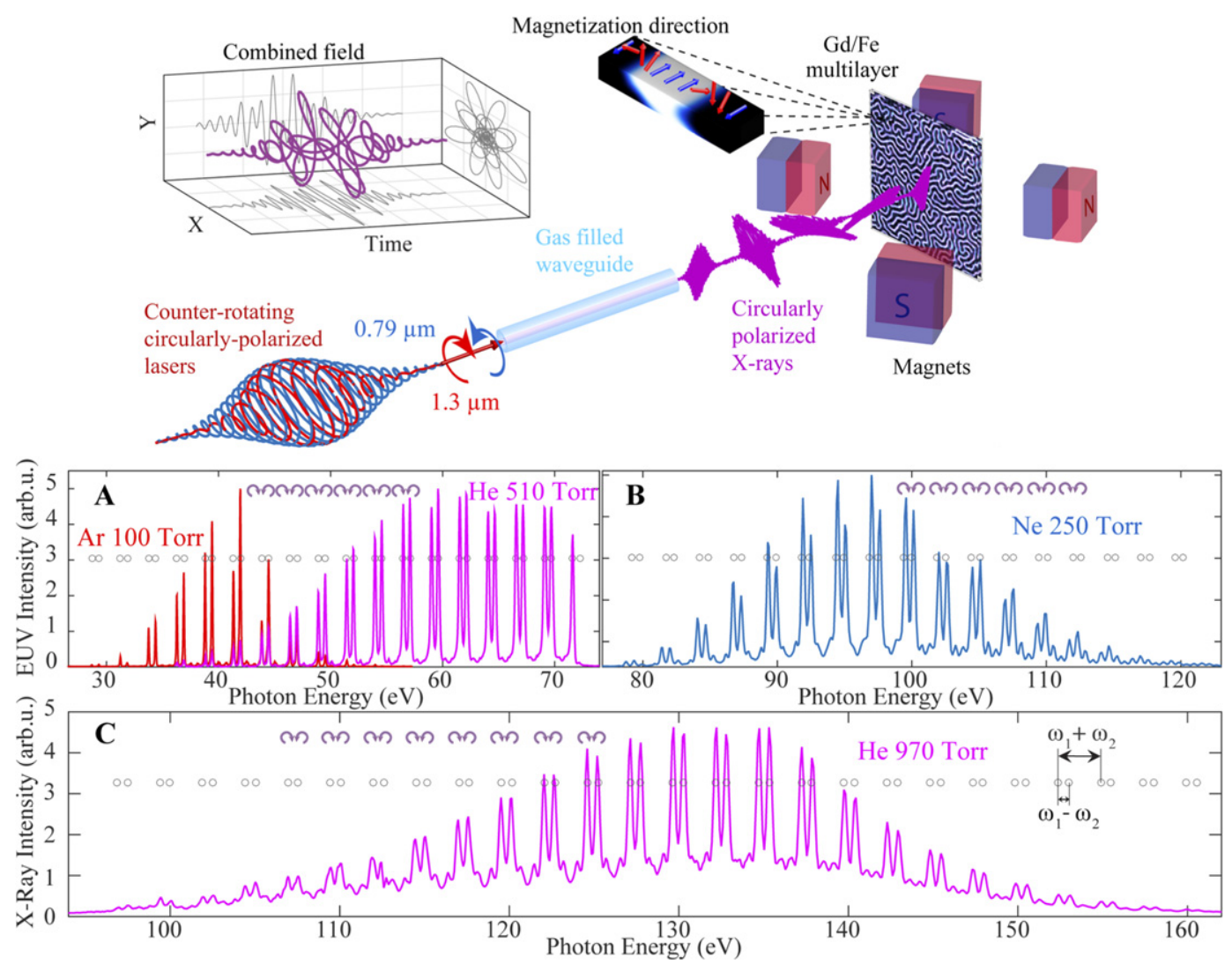

Figure 2. Setup for producing bright circularly polarized high-order harmonics. (top) HHG driven by a two-color counter-rotating circularly polarized field can be well phase matched, and leads to the generation of pairs of circularly polarized harmonics with opposite helicity due to SAM conservation rules. (bottom) Circularly polarized EUV and soft x-ray HHG. Experimental HHG spectra generated from Ar (A), Ne (B), and $\mathrm{He}(\mathrm{A})$ and $(\mathrm{C})$ driven by counterrotating 0.79 and $1.3 \mu \mathrm{m}$ laser fields. All spectra show a peak-pair structure, located at positions predicted by energy and SAM conservation. The separation within each pair is $\omega_{1}-\omega_{2}$, and different pairs are separated by $\omega_{1}+\omega_{2}$. Reproduced with permission from [25].

angular momentum properties. Light beams can exhibit two degrees of freedom associated with their angular momentum: spin angular momentum (SAM), indicating the direction in which the electric field oscillates in time, or polarization; and OAM related to the spatial profile of the phase or the wavefront of the electric field. SAM is a microscopic property of light fields, characterized by the spin, $\sigma= \pm 1$, and defining the light field polarization, from right $(\sigma=1)$ or left $(\sigma=-1)$ circularly polarized, to elliptically or linearly polarized depending on the photon spin combination. On the other hand, OAM is related to the transverse spiral-phase structure of a light beam [52], representing a macroscopic property that is characterized by the topological charge, $\ell$ - the number of $2 \pi$-phase shifts along the azimuth. Remarkably, while the spin is restricted to \pm 1 , the topological charge can take infinite discrete values.

Angular momentum can be imprinted onto visible/IR light beams using waveplates, or spatial light modulators, among other techniques. However, this becomes considerably more challenging for EUV and x-ray beams, while also being highly inefficient [53]. During the last decade, a new set of powerful approaches have been developed to imprint and control the SAM and OAM content of HHG beams in the EUV/SXR regimes through a change of paradigm - instead of imprinting angular momentum into a short wavelength beam using x-ray optics, we can harness the extreme nonlinear process of HHG to up-convert the angular momentum properties of the driving lasers. However, this is far from trivial. Fortunately, over the past five years, several different approaches have succeeded in precisely imprinting SAM and/or OAM into HHG beams.

Ultrafast pulses of linearly and circularly polarized light make it possible to capture the fastest spin dynamics in materials and to uncover a wealth of new fundamental understanding about spin scattering and transport. For a long time, it was thought that HHG could only produce linearly polarized harmonics, due to the weak rescattering/recollision efficiency when driven by circularly polarized IR pulses - where the recolliding electron misses the parent ion when driven by a circularly polarized laser field [54]. However, several techniques have recently succeeded in generating bright circularly polarized harmonics, making use of sophisticated combinations of driving laser fields $[55,56]$, together with optimally phase matched geometries [24, 25, 27].

Among these techniques, HHG driven by a two-color counter-rotating circularly polarized field-a bi-circular field - is the most efficient method for generating circularly polarized harmonics, with brightness and energies comparable to one-color linearly polarized HHG (see figure 2). 
(a)

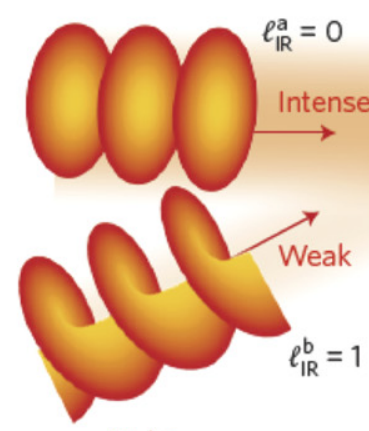

IR drivers

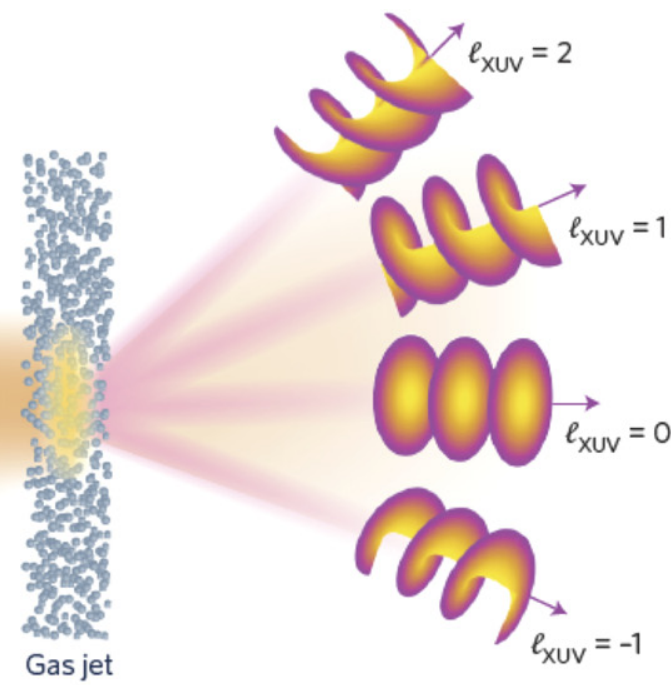

Single-OAM XUV harmonics

(b)

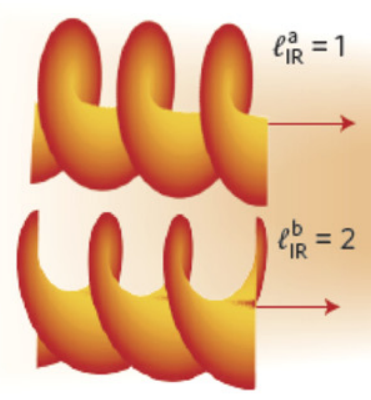

IR drivers

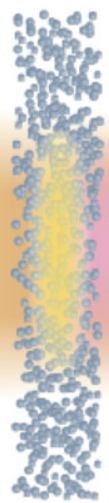

Gas jet

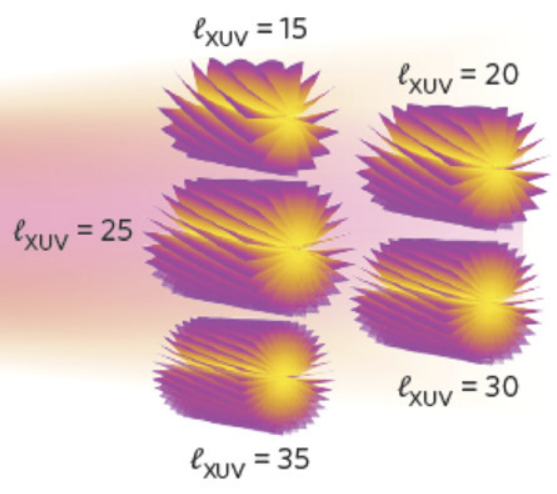

Multiple-OAM XUV harmonics (c) Scheme for the generation of self-torqued beams

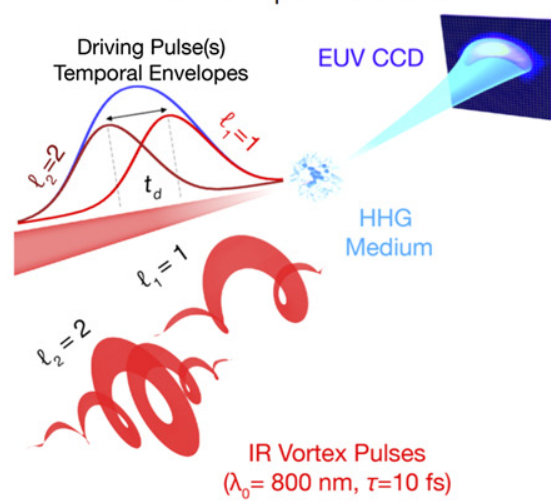

Temporal evolution of the OAM

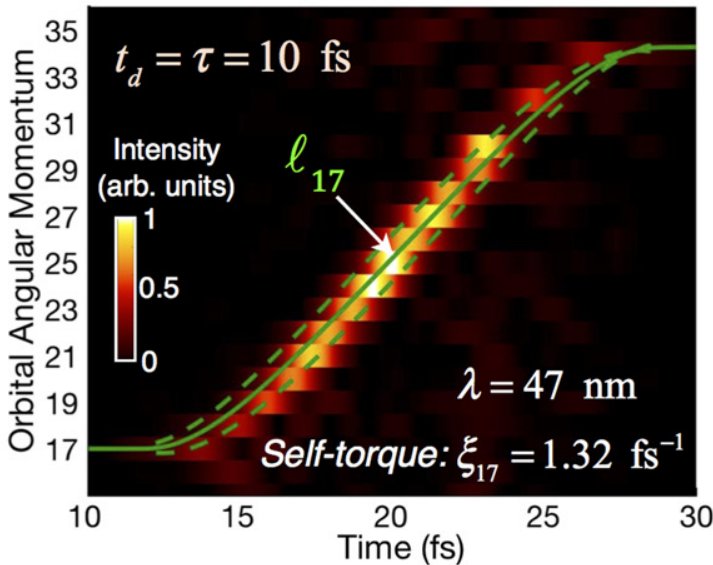

Figure 3. Generation of high-order harmonics with custom OAM properties. (a) HHG driven by a non-collinear combination of vortex beams with different OAM leads to the generation of a fan of harmonic vortices with custom OAM [65, 66]. (b) HHG driven by a collinear superposition of vortex beams with different OAM leads to the generation of harmonic beams with broad OAM content [67]. (c) The use of two time-delayed vortex driving fields allows for the generation of high-order harmonics with self-torque, i.e., with time-dependent OAM, as indicated in the right panel [31]. Panels (a) and (b) are reprinted by permission from Springer Nature Customer Service Centre GmbH: Springer Nature, Nature Physics [61] 2017. Panel (c) is from [31]. Reprinted with permission from AAAS. 
Remarkably, HHG from single atoms driven by a bi-circular field was theoretically proposed two decades ago $[55,56]$. Optimal phase matching was recently experimentally demonstrated in a waveguide geometry [24, 25, 27]—bright, stable, HHG is produced, since the combined two-color field is not sensitive to the inevitable phase slip that occurs when the light of different colors propagates in a medium. This is stark contrast to HHG driven by two-color linearly-polarized laser fields. The primary spectral feature of bi-circular HHG is the suppression of harmonic orders that do not conserve SAM. As a consequence, the HHG spectra consists of pairs of harmonics with opposite helicity [i.e., with left-circular (LCP, $\sigma=-1$ ) or right-circular polarization (RCP, $\sigma=1$ ), see figure 2]. The generation of both LCP and RCP in bi-circular HHG results in the emission of linearly polarized attosecond pulses, where the polarization direction rotates from pulse to pulse. Different modifications of this technique demonstrated the possibility to generate elliptically polarized attosecond pulses.

More recently, other approaches have implemented to generate circularly polarized harmonics. For example, the use of non-collinear counter-rotating driving beams with the same color results in a linearly polarized field at the focus, with varying polarization direction along the transverse plane-thus allowing for high harmonics to be generated at each local position. Upon propagation to the far-field, the macroscopic superposition results in the generation of two spatially separated circularly polarized harmonic beams, allowing for the generation of attosecond pulses with controllable ellipticity [26, 28, 30]. This approach also has the advantage of being amenable to generating isolated circularly polarized attosecond pulses [28]. Other techniques that have successfully generated elliptical to circularly polarized harmonics include the use of two HHG sources through the use of two orthogonally polarized foci [50] or two different gas jets [57].

There are also scenarios where the state of polarization or SAM varies from point to point of a light beam, conforming to so-called vector beams. Recently HHG has been used to generate vector beams well beyond the visible region, through the use of waveplates or conical refraction beams [58, 59].

HHG also represents a unique opportunity to transfer the OAM of the driving laser field onto shorter-wavelength beams, circumventing the limitations of other approaches that imprint OAM directly into EUV/SXR [60, 61]. One of the first experiments to explore OAM-HHG pioneered the idea of driving HHG with an IR vortex beam with $\ell_{\mathrm{IR}}=1$ [62]. These initial results were unclear, as they reported the generation of harmonic vortices with $\ell_{\mathrm{EUV}}=1$, i.e. with the same topological charge as that of the fundamental driving field, thus breaking the expected OAM conservation rules in the HHG process. However, theoretical simulations predicted that when HHG is driven by an IR vortex with topological charge $\ell_{\mathrm{IR}}$, the OAM of the $q$ th-order harmonic follows the simple rule $\ell_{q}=q \ell_{\mathrm{IR}}$ [63]. This scaling reflects the OAM conservation in the conversion of $q$ IR-photons into a $q$ th-order harmonic. Shortly afterward, different experiments confirmed the conservation of OAM in the HHG process, and thus the generation of high-order harmonics with OAM. In the temporal domain, this frequency comb of harmonic vortices with $\ell_{\mathrm{q}}=q \ell_{\mathrm{IR}}$ can be synthesized into helical attosecond beams, as predicted in 2013 [63], and measured in 2016 [64]. Helical attosecond beams convey a unique spatio-temporal light structure to produce attosecond electron springs through photoionization.

On the other hand, if HHG is driven by a combination of two IR vortices with different $\mathrm{OAM}, \ell_{\mathrm{IR}, 1}$ and $\ell_{\mathrm{IR}, 2}$, an even richer scenario for the generation of harmonic vortices with custom OAM appears. If the two drivers are combined in a noncollinear configuration, different processes of sum and difference frequency mixing from the two driving beams, together with momentum conservation, resulting in the production of a fan of spatially separated EUV beams with different topological charge $[65,66]$. If the two drivers are combined in a collinear scheme, each harmonic spans into a distribution of OAM, given by $\ell_{q}=n_{1} \ell_{\mathrm{IR}, 1}+n_{2} \ell_{\mathrm{IR}, 1}$ [67], where $n_{1}$ and $n_{2}$ are the number of photons absorbed from each driver $\left(n_{1}+\right.$ $\left.n_{2}=\mathrm{q}\right)$. The central topological charge of the OAM distribution depends on the intensity of each driver, which reflects the sensitivity of HHG with the purity of the OAM-mode of the driving beam. Recently, the unique mapping opportunity that offers HHG driven by OAM pulses, has resulted in the emission of light beams with self-torque, a property associated with its temporal variation of the OAM [31]. By temporally delaying two driving pulses with different topological charges in a collinear configuration, HHG naturally emits harmonic pulses whose topological charge varies continuously in time. This recent finding has opened the possibility to sculpt laser pulses with varying OAM, providing a new tool for exploring the dynamics of topological laser-matter interaction (figure 3).

Finally, high harmonic vortices with both SAM and OAM structures can now be generated. In particular, the use of a two-color counter-rotating circularly polarized vortex can efficiently generate pairs of circularly polarized harmonic vortex with opposite helicity. Moreover, by a proper combination of the OAM of the drivers, attosecond pulse trains with custom polarization, from linear to circular can be obtained [29].

\subsection{Summary}

The extreme nonlinear process of HHG enables control over $\mathrm{X}$-ray light at an unprecedented level—on sub-attosecond temporal and sub- $\AA$ spatial scales. Harnessed the extreme nonlinear process of HHG to demonstrate. When combined with record sub-wavelength imaging (see section 5), this capability may finally make it possible to bring super-resolution methods into the x-ray region for a host of critical nanotechnologies. The discovery of a new property of light beams-the self-torque, associated to a time-varying OAM — may provide routes for dynamic imaging of attosecond chiral dynamics (see section 3): short wavelength OAM-SAM beams are also ideal for launching, manipulating, and detecting nanoscale chiral textures for applications in sensing, metrology, energyefficient logic/memory devices [68]. Indeed, recent theories have shown that SAM and OAM can be exploited for nanospectroscopies with enhanced contrast $[69,70]$. 

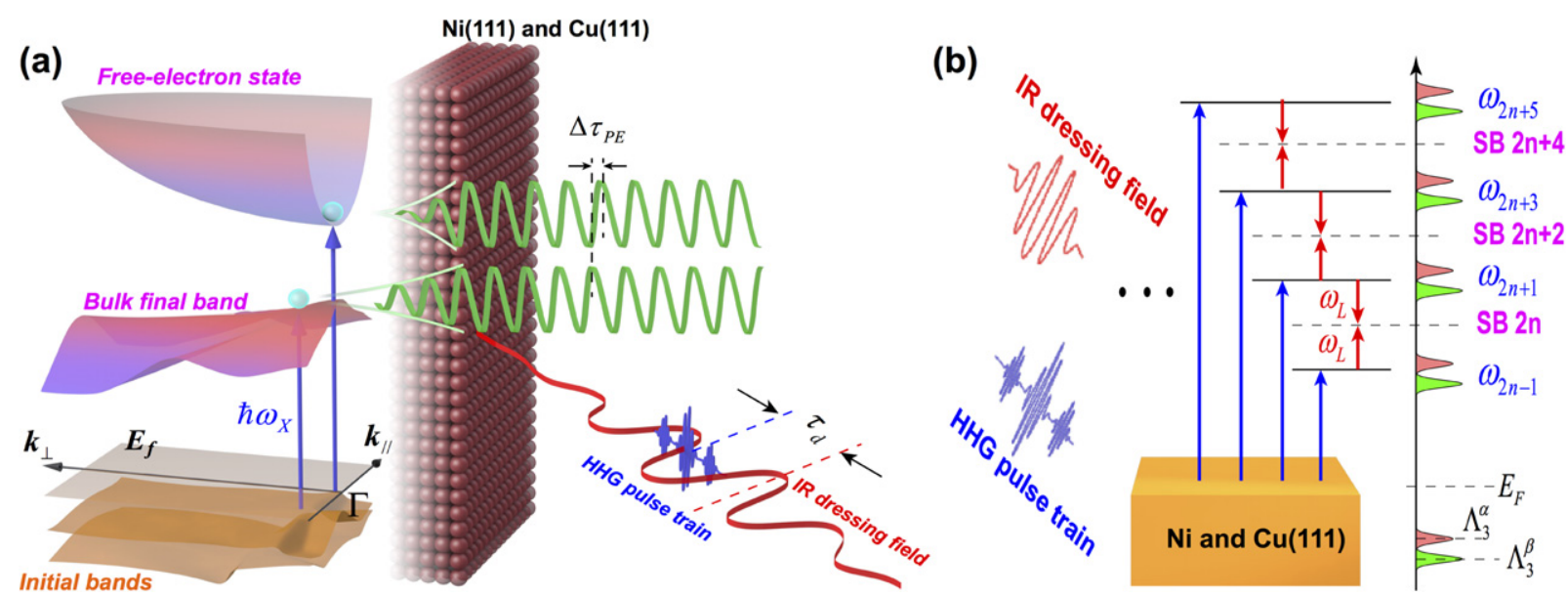

Figure 4. (a) Illustration of the atto-ARPES setup. Different initial and final states can be accessed by different HHG photon energies and polarizations. An IR dressing field is applied to modulate the photoelectron spectrum via laser-assisted photoemission. (b) Illustration of the resulting quantum-path interferences (RABBITT). Panel (a) is from [33]. Reprinted with permission from AAAS. Panel (b) is reproduced with permission from [34].

\section{Probing intrinsic attosecond dynamics in materials using high harmonic ARPES}

\subsection{Introduction}

The ability to generate bright attosecond pulses and pulse trains through HHG process has made it possible to capture the fastest coupled charge, spin, phonon, and transport dynamics in a host of atomic, molecular, materials and nano systems. However, to probe intrinsic attosecond dynamics, both the energy scale of the interactions and dynamics, and the spectroscopy method used, need to be well matched. Processes such as optical excitation of charges and spins [35-37], electron momentum and energy redistribution, electron-electron scattering and screening and highly-excited electron dynamics $[33,34]$ can all support intrinsic attosecond dynamics, that are now being revealed using HHG probes. Moreover, in the strongly-coupled materials that support such attosecond dynamics, these processes all depend on the electronic band structure. Hence the challenge-how to achieve attosecond time resolution while maintaining the $\sim 100 \mathrm{meV}$ energy resolution so that band-specific dynamics can be extracted?

In many of initial experiments, the use of isolated attosecond pulses to achieve high time resolution was (necessarily) accompanied by a broad EUV bandwidth. When measuring a quantity such as the timing of photoemission, this approach yields an integrated broadband photoemission feature that averages over multiple valence bands [71, 72]. To overcome this challenge and measure photoemission from individual valence bands, we combined attosecond pulse trains of well-defined harmonics, with laser-assisted photoemission [73], and ARPES. This new technique we call atto-ARPES can measure and distinguish photoemission time delays from individual valence bands into either free-electron final states, or into final states corresponding to unoccupied bulk bands of transition metals such as $\mathrm{Ni}(111)$ and $\mathrm{Cu}(111)$ [33, 34]. This is critical for a full understanding of the physics and to compare theory and experiment. For isolated atoms, it is generally agreed that the photoelectron time delay arises from a convolution of a Wigner-like time delay due to scattering in the atomic potential and a measurement-induced delay due to propagation of photoelectrons in the laser field [74]. Unlike isolated atoms, however, the interpretation of a photoemission time delay in quantum materials is much more complicated, owing to the presence of complex band structures and many-body interactions [71, 72, 75-77]. Thus, techniques such as atto-ARPES described below, which can associate the time delay with the material band structure, can accurately and sensitively probe how the intrinsic properties of the material influence electron interactions.

\subsection{Description of the atto-ARPES technique}

The schematic of the atto-ARPES setup is shown in figure 4. High harmonics covering a wide range of EUV photon energies are generated by focusing a strong laser field into different noble gas targets (xenon, krypton and argon). The polarization of the EUV HHG beam can be adjusted by using a halfwave plate, which allows us to harness the selection rules to selectively excite photoelectrons from different initial states (bands) and into different final states. The harmonics, which consist of a train of $\sim 400$ attosecond pulses separated by $\sim 1.5 \mathrm{fs}$, are then focused onto an atomically clean single-crystal metal surface $[\mathrm{Ni}(111)$ and $\mathrm{Cu}(111)]$. The resulting photoelectron spectrum is then collected by an angle-resolved detector. A weak IR beam with $p$ polarization propagates collinearly with the EUV field, and is used to modulate (i.e. dress) photoelectron spectrum to extract the band and final state dependence of the photoemission time. This 'laser-assisted photoemission' from surfaces was first used to measure the core level lifetime of adsorbates on surfaces [73, 78].

As shown in figure 4(b), in the presence of several harmonics spectrally (i.e. attosecond pulse trains in time) and the IR dressing laser field, there exist two distinct quantum paths from an initial state to the same sideband, namely absorption of a harmonic with simultaneous absorption/emission of an IR 

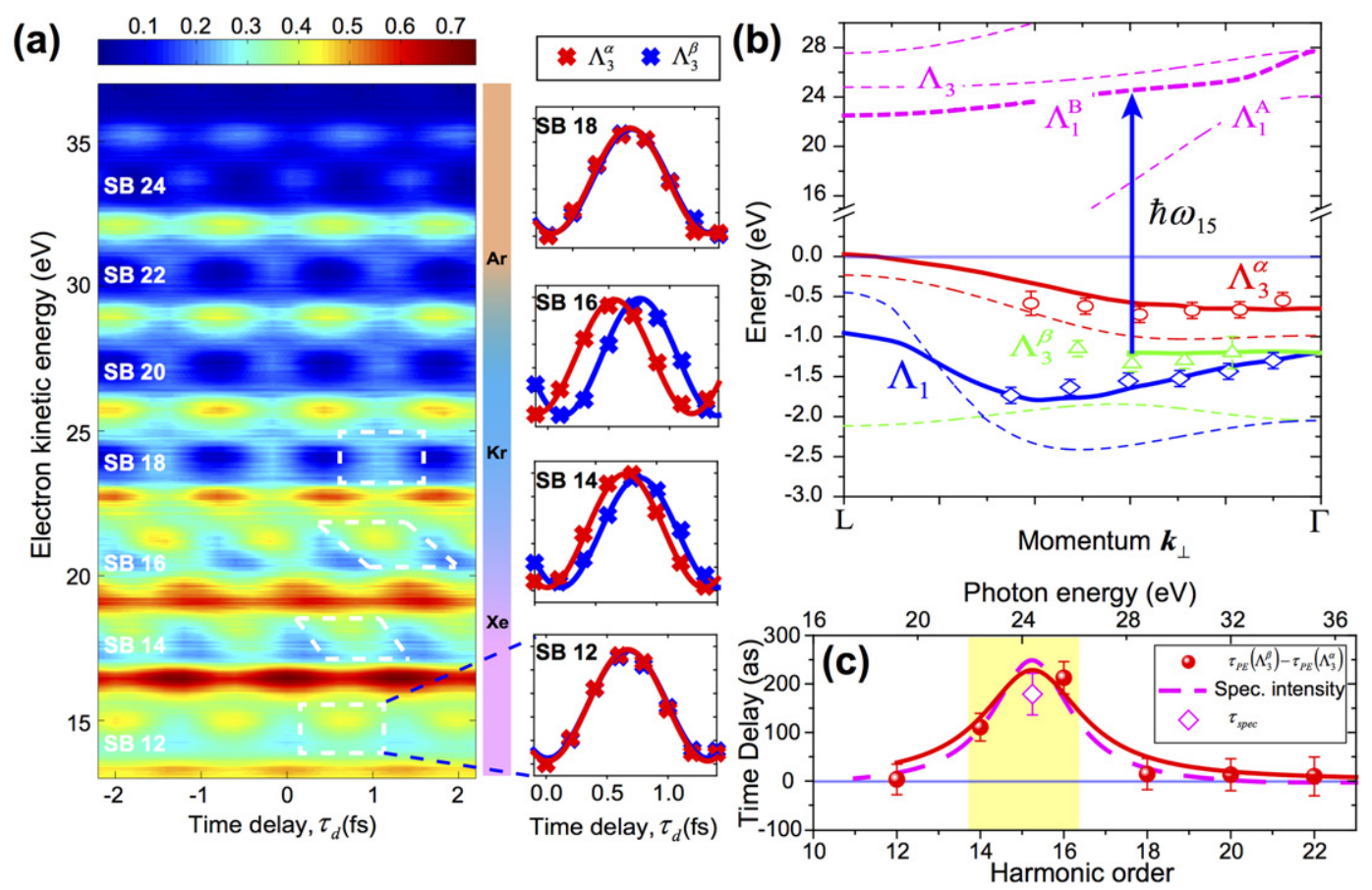

Figure 5. (a) Two-dimensional map of the photoelectron yield as a function of photoelectron energy and pump-probe delay $\tau_{\mathrm{d}}$ excited by $s$-polarized HHG. Right: 1D lineouts for photoemission from the $\Lambda_{3}{ }^{\alpha}$ and $\Lambda_{3}{ }^{\beta}$ states. (b) Band structure along the $\Gamma-L$ direction of Ni(111). The solid lines are the results of previous experiments [82]. The dashed lines are the results of density functional theory calculations. (c) Photoemission time delay $\tau_{\mathrm{PE}}\left(\Lambda_{3}^{\beta}\right)-\tau_{\mathrm{PE}}\left(\Lambda_{3}^{\alpha}\right)$ as a function of harmonic order and photon energy. The dashed line is the spectral intensity variation of photoelectrons from the $\Lambda_{3}{ }^{\beta}$ band. The open diamond symbol is the photoelectron lifetime obtained from the spectral analysis. From [33]. Reprinted with permission from AAAS.

laser photon: (i) $\hbar \omega_{2 n-1}+\hbar \omega_{L}$ or (ii) $\hbar \omega_{2 n+1}-\hbar \omega_{L}$. When the relative time delay $\tau_{\mathrm{d}}$ between the HHG pump and the IR probe is changed, the intensity of sidebands [73] with a photon energy $\hbar \omega_{2 n}$ is modulated $[79,80]$ as a result of quantum-path interference:

$$
S_{2 n}\left(\tau_{\mathrm{d}}\right)=A_{0} \sin \left[2 \omega_{L}\left(\tau_{\mathrm{d}}-\tau_{X}-\tau_{\mathrm{PE}}\right)\right]
$$

where $A_{0}$ is the intensity of modulation, $\tau_{X}=$ $\left(\phi_{2 n+1}-\phi_{2 n-1}\right) / 2 \omega_{L}$ represents a time delay induced by the phase chirp between neighboring harmonic orders, and $\tau_{\mathrm{PE}}$ is the delay time associate with the photoemission process. By simultaneously measuring photoelectron wavepackets from different initial states excited by the same harmonic orders, we can also cancel the influence of attochirp $\left(\tau_{X}\right)$. Such quantum interfering pathways was first implemented for isolated atoms, using a technique called RABBIT (the reconstruction of attosecond beating by interference of two-photon transition) [79].

\subsection{Influence of material band structure on photoemission: attosecond photoelectron lifetimes}

To extract the photoemission time delay from a Ni(111) surface for different initial and final states, in figure 5(a) we plot a set of interferograms that consist of the photoelectron peaks from $s$-polarized high harmonics, together with their laserassisted sidebands [33]. These data were integrated over a small angle $\left( \pm 2.5^{\circ}\right)$ around the $\Gamma$ point. Because of the photoemission selection rules [81], only the two valence bands with the $\Lambda_{3}$ symmetry are excited by $s$-polarized HHG fields, as shown in figure 5(b). A notable feature is that photoelectrons from the $\Lambda_{3}^{\beta}$ band are significantly delayed compared to those emerging from the $\Lambda_{3}^{\alpha}$ band for sideband (SB) 16. More interestingly, this time delay gradually vanishes at increasing and decreasing photon energies, showing a nonmonotonic change of $\tau_{\mathrm{PE}}\left(\Lambda_{3}^{\beta}\right)-\tau_{\mathrm{PE}}\left(\Lambda_{3}^{\alpha}\right)$. The time delay results are summarized in figure 5(c). The maximum of time delay $\tau_{\mathrm{PE}}\left(\Lambda_{3}^{\beta}\right)-$ $\tau_{\mathrm{PE}}\left(\Lambda_{3}^{\alpha}\right)$ coincides with the spectral intensity resonance as shown in figure 5(c), strongly indicating that the observed photoemission time delay originates from the resonant interband transition between the low energy $\Lambda_{3}^{\beta}$ band and the unoccupied $\Lambda_{1}^{B}$ band (figure 5(b)). More importantly, the time delay measured using laser-assisted photoemission, $\tau_{\text {chron }}=212 \pm 30$ as, agrees (within error bars) with the value from the spectral resonance, $\tau_{\text {spec }}=179 \pm 43$ as, indicating the direct timedomain measurement of photoelectron lifetime on attosecond timescales.

Other important evidence that the band structure of the material strongly influences the photoemission time can be seen from the angle-dependence of the photoelectron time delay. Here, the use of a hemispherical analyzer allows us to extract how the photoemission time depends on the transverse momentum of the electron $\boldsymbol{k}_{/ /}$. In figures 6(a) and (b), we plot the angle-dependence of $\tau_{\mathrm{PE}}\left(\Lambda_{3}^{\beta}, \theta\right)-\tau_{\mathrm{PE}}\left(\Lambda_{3}^{\alpha}, \theta\right)$ for SB 16 
(a) Electron momentum, $\boldsymbol{k}_{/ /}\left(\AA^{-1}\right)$

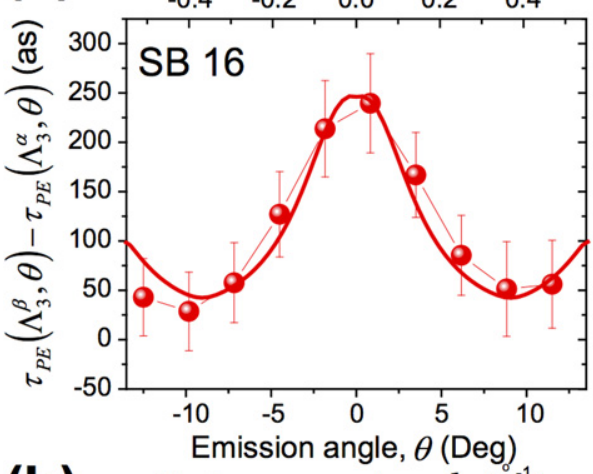

(b) Electron momentum, $\boldsymbol{k}_{/ /}\left(\AA^{-1}\right)$

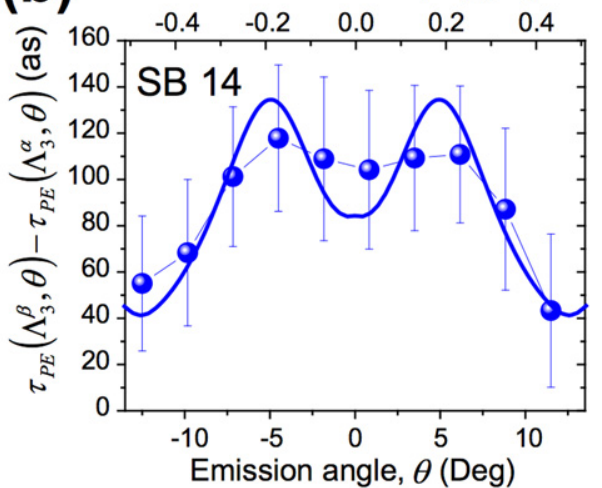

(c)

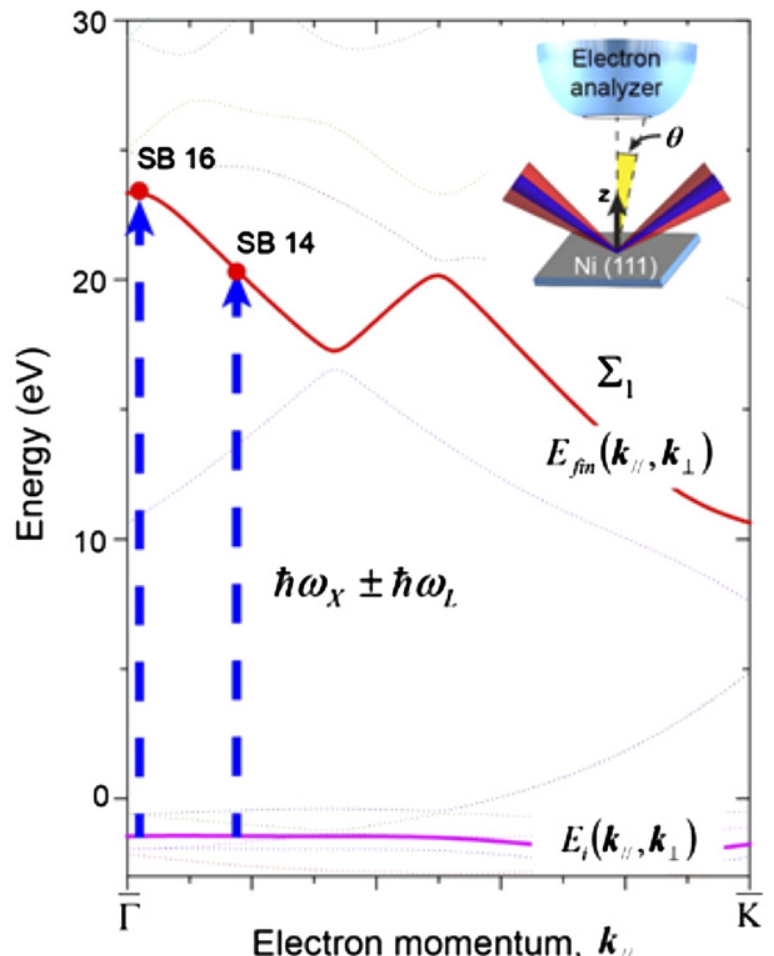

Figure 6. (a) and (b) angle-dependent photoemission time delay of $\tau_{\mathrm{PE}}\left(\Lambda_{3}^{\beta}, \theta\right)-\tau_{\mathrm{PE}}\left(\Lambda_{3}^{\alpha}, \theta\right)$ from $\mathrm{Ni}(111)$ for the SB 16 and SB 14 respectively. The solid lines are the results of our analytical model. (c) Illustration of the direction transitions in the $\boldsymbol{k}_{/ /}$direction for the SB 14 and SB 16. From [33]. Reprinted with permission from AAAS.

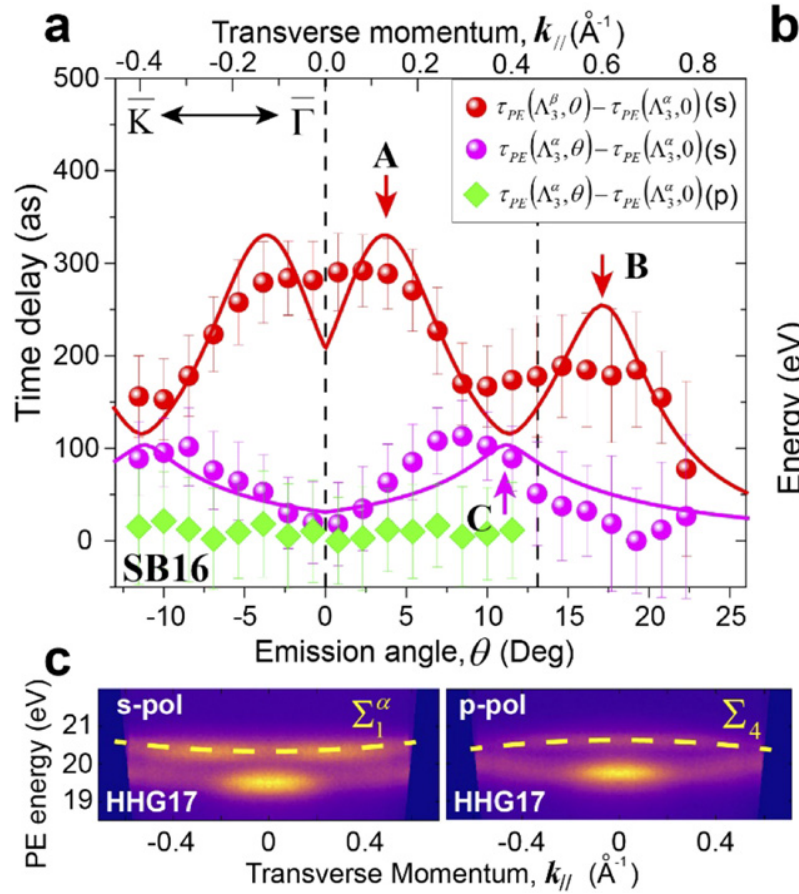

b

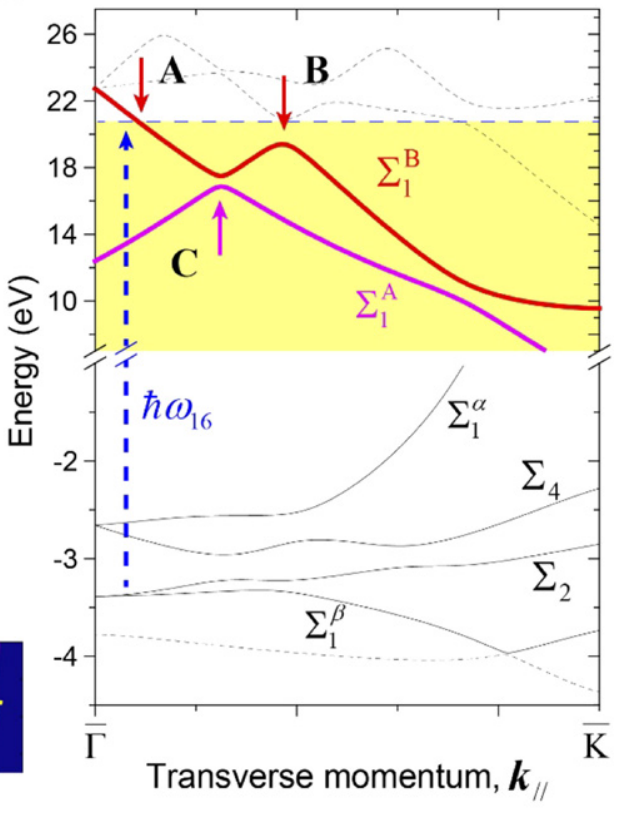

Figure 7. (a) Results of angle-dependent photoemission time delays for $\mathrm{SB} 16$ from $\mathrm{Cu}(111)$ with different initial bands and different polarizations. (b) Illustration of the band structure of $\mathrm{Cu}(111)$ along the $\boldsymbol{k}_{/ /}$direction. The features of band dispersion are labeled, in direct correspondence to the features in the experimental data in (a). (c) Static photoelectron spectra expected by $s$ - and $p$-polarized EUV fields. Different dispersion of the bands is highlighted by the dashed lines. 

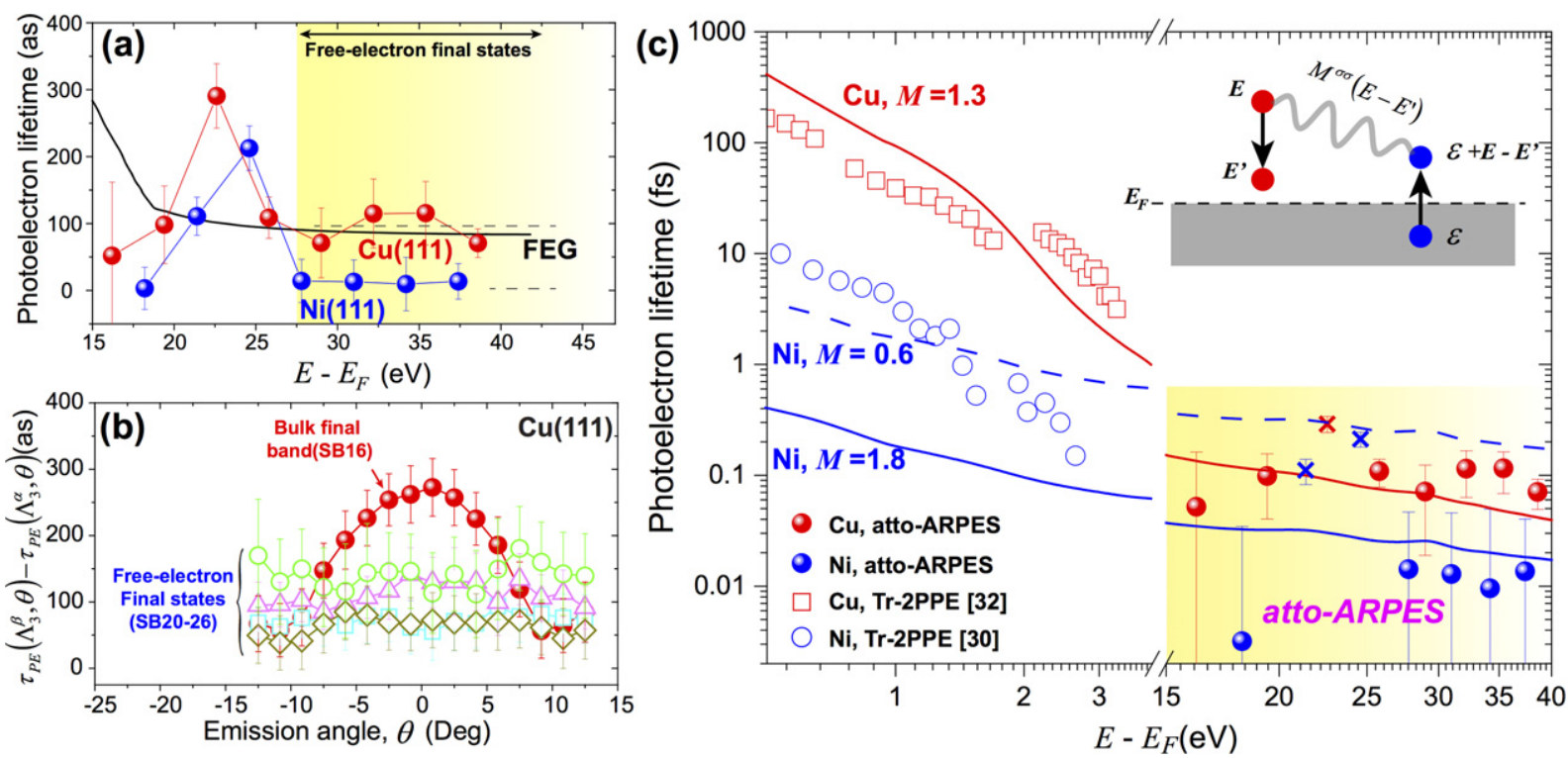

Figure 8. (a) Comparison between the photoelectron lifetime from the same initial band ( $\Lambda_{3}^{\beta}$ ) from the $\mathrm{Cu}(111)$ and $\mathrm{Ni}(111)$ surfaces. (b) Angle-dependent photoemission time delay of $\tau_{\mathrm{PE}}\left(\Lambda_{3}^{\beta}, \theta\right)-\tau_{\mathrm{PE}}\left(\Lambda_{3}^{\alpha}, \theta\right)$ measured on $\mathrm{Cu}(111)$, which clearly shows the difference between a resonant transition to a bulk final band (SB 16) and those to free-electron final states. (c) Comparison between the model of Fermi's golden rule and the experimentally measured lifetime. The low-energy data $(0.5-3 \mathrm{eV})$ are measured using $\mathrm{Tr}-2 \mathrm{PPE}$ method for $\mathrm{Cu}$ [87] and for $\mathrm{Ni}$ [86]. The experimentally measured low-energy electron lifetime approaches the bare electron-electron scattering limit (solid blue line, $M=$ 1.8 for $\mathrm{Ni}$ ) at an energy $E_{\text {crit }} \sim 3 \mathrm{eV}$. Inset: illustration of the electron-electron scattering process described by equation (2). Reproduced with permission from [34].

and SB 14. We find that the relative time delay between photoelectrons from two different valence bands reaches a maximum value of $\sim 220$ as at the $\bar{\Gamma}$ point $(\theta=0)$ - whereas it reduces to $\sim 30$ as when emission angle approaches $\theta= \pm 15^{\circ}$. The overall trend is symmetric around the $\bar{\Gamma}$ point, strongly indicating the band-structure origin of the observed time delay.

More interestingly, the photoemission delay $\tau_{\mathrm{PE}}\left(\Lambda_{3}^{\beta}, \theta\right)-$ $\tau_{\mathrm{PE}}\left(\Lambda_{3}^{\alpha}, \theta\right)$ exhibits a double-peak structure for SB 14 . This angle-dependent time delay can be quantitatively reproduced by our analytical model [33], taking into account the dispersion of the $\Sigma_{1}$ final band along the $\boldsymbol{k}_{/ /}$direction, as shown in figures 6(a) and (b). For SB 16, the photon energy is high enough to excite the interband transition at the $\bar{\Gamma}$ point, while the time delay monotonically decreases at larger emission angles, since final-state band dispersion moves this transition off resonance. On the other hand, for SB 14, the resonant transition is not accessible at the $\bar{\Gamma}$ point, but then moves on resonance and yields a maximum time delay at $\sim 6^{\circ}$ emission angle (figure 6(b)).

Similar angle-resolved photoemission time delays can also be seen on a $\mathrm{Cu}(111)$ surface for the $\Lambda_{3}^{\beta}$ and $\Lambda_{3}^{\alpha}$ initial bands, as summarized in figure 7(a). In addition to the interband resonance between the low-energy $\Lambda_{3}^{\beta}$ ( $\Sigma_{1}^{\beta}$ in the $\boldsymbol{k}_{/ /}$direction) band to the unoccupied $\Lambda_{1}^{B}\left(\Sigma_{1}^{B}\right)$ final band, we also observe clear evidence of the coupling between the $\Lambda_{3}^{\alpha}\left(\Sigma_{1}^{\alpha}\right)$ band and the $\Lambda_{1}^{A}\left(\Sigma_{1}^{A}\right)$ final band. The variation of attosecond delays as a function of $\boldsymbol{k}_{/ /}$is in good agreement with the dispersion of the final band. More interestingly, the angle-dependence of the attosecond time delay $\Lambda_{3}^{\alpha}\left(\Sigma_{1}^{\alpha}\right)$ disappears when the polarization of the EUV field is changed to $p$-polarization. Correspondingly, there is a change of band dispersion in the static photoelectron spectra, as shown in figure 7(c), which indicates photoelectrons from a different initial state $\left(\Sigma_{4}\right)$ are excited by p-polarized EUV fields. Our results clearly show that bandstructure effects can significantly influence the energy-, angleand polarization-dependent photoemission time.

\subsection{Distinguishing attosecond electron-electron screening and scattering}

In figure $8(\mathrm{a})$, we plot the attosecond photoemission time delay measured from the same valence band $\left(\Lambda_{3}^{\beta}\right)$ for both $\mathrm{Ni}(111)$ and $\mathrm{Cu}(111)$. Here, we find that the photoemission time for free-electron final states (i.e. off resonance) from $\mathrm{Cu}(111)$ is generally $\sim 100$ as longer than that from $\mathrm{Ni}(111)$. This 100 as time difference cannot be explained by another final-state resonance because we did not observe any photoelectron yield enhancement in this energy range. Moreover, it exhibits little momentum (angle) dispersion - unlike the behavior observed when the final state is on resonance with a high-energy unoccupied state (figure 8(b)). In figure 8(a), we compare our measured photoelectron lifetime of $\mathrm{Cu}$ to the free-electron gas (FEG) model [83]. Interestingly, we find that the FEG model matches the off-resonance (i.e., free-electron final-state) lifetime of $\mathrm{Cu}(111)$ very well, but not for $\mathrm{Ni}(111)$, which is $\sim 100$ as shorter. Note that this trend is very different from the hotelectron lifetimes measured at low energy ( $<3 \mathrm{eV}$ above $\left.E_{\mathrm{F}}\right)$, which exhibit a strong deviation from the FEG model due to the presence of $d$-band screening [84, 85]. Our results indicate 
that the electron screening does not have a strong influence on the photoelectron lifetime when the final state energy is higher than $20 \mathrm{eV}$.

Interestingly, we find that the $\sim 100$ as lifetime difference can be attributed to differences in the band structure of $\mathrm{Cu}$ and $\mathrm{Ni}$, which results in different electron-electron scattering rates between photoelectrons and other electrons in the conduction bands. The electron-electron scattering process is illustrated in the inset of figure 8(c). The scattering rate can be calculated from Fermi's golden rule in the random- $\boldsymbol{k}$ approximation [34, $86]$, and the results are plotted in figure 8(c), in direct comparison with the experimentally measured lifetime of photoexcited electron from 0.5 to $40 \mathrm{eV}$. The low-energy results were reproduced from time-resolve two-photon photoemission ( $\mathrm{Tr}-$ 2PPE) measurements [86,87]. Thus, the photoexcited-electron lifetime in $\mathrm{Cu}$ can be well explained by a mostly energyindependent Coulomb matrix element for electron-electron scattering, while there is a strong energy dependence of the Coulomb matrix element in Ni. There, $M=1.8$ is the best fit for high-energy photoelectrons and is close to that observed in $\mathrm{Cu}$, whereas $M=0.6$ is best for low-energy photoelectrons. As shown in figure 8(c), the measured electron lifetime at low energy in $\mathrm{Ni}$ is more than one order of magnitude longer than the bare electron-electron scattering limit, indicating strongly enhanced screening in this energy range, possibly due to the high density of states of Ni at the Fermi energy [86].

\subsection{Summary}

By combining attosecond pulse trains generated by the HHG process and ARPES techniques, we investigated photoemission timescales from transition metals, $\mathrm{Ni}$ and $\mathrm{Cu}$. From our results, we demonstrate that atto-ARPES can directly measure photoelectron lifetimes in the time domain. Moreover, we unambiguously show that the material band structure has a strong influence on the photoemission time-it is dependent on the photon/photoelectron energy, emission angle and light polarization. By comparing photoelectron lifetimes from different materials, we show that strong electron-electron scattering in the unfilled $d$ band of $\mathrm{Ni}$ decreases the lifetime of photoelectrons by $\sim 100$ as relative to the photoelectrons emitted from the same band of $\mathrm{Cu}$. Our results represent the first time domain study of electron-electron interaction on attosecond timescales, and represents a new technique and a new perspective on the broader relevance of attosecond science.

\section{New understanding of electron-phonon couplings and phase transitions via ultrafast electron calorimetry}

\subsection{Introduction to ultrafast electron calorimetry}

The ability to probe the full dynamic response of quantum materials on the length- and time-scales ( $\AA$ to attoseconds on up) fundamental to charge, spin and phonon interactions is leading to a host of new discoveries. The coupled interactions between charges, spins, orbital and lattice degrees of freedom are key to determining the state of a material, whether metallic, insulating, superconducting or magnetic [88-90]. Under thermal equilibrium conditions these states can be tuned by varying the temperature, pressure, chemical doping or dimensionality. However, their inherent complexity and rich phase landscape make them challenging to understand or manipulate deterministically.

Fortunately, the development of ultrashort coherent light sources from the IR to $\mathrm{x}$-ray region enables a powerful new set of ultrafast spectroscopies that can uniquely address these challenges [14, 38, 39, 73, 91-119]. In the past, exciting a material with an ultrafast laser was usually thought to create a highly out-of-equilibrium state. By using high harmonic (HHG) sources to probe the full dynamic band structure and electron temperature, we have been able to very delicately excite and probe strongly-coupled materials - allowing us to link their dynamic response to their equilibrium properties, to drive materials into new metastable states that cannot be reached under equilibrium conditions, and to uncover a new understanding of excited states and phase transitions.

The key to this ability is a new approach we call ultrafast electron calorimetry [35, 36, 38, 39]. Using HHGbased time- and angle-resolved photoemission spectroscopy (trARPES), we can measure the full electronic band structure and extract the dynamic electron temperature. Electrons react very quickly upon excitation by light, on attosecond to femtosecond timescales. Moreover, their heat capacity is small compared to the total heat capacity of the material. After excitation by the laser, except for the case of resonantly excited modes [113-119], the excited electrons could rapidly establish a hot Fermi-Dirac distribution within 20-50 fs in the materials with strong electron-electron scattering. The energy then gradually flows from the hot electrons to the phonon bath on a few ps timescales. This relatively high decoupling of excitations and interactions in the time domain means that by measuring the dynamic electron temperature $\left(T_{\mathrm{e}}\right)$, we can sensitively detect changes in the microscopic interactions within a material, such as electron-phonon couplings or phase changes - that are indicated by either a change in slope of, or jump in, the electron temperature as a function of excitation laser fluence. When coupled with the ability of HHG trARPES to simultaneously capture the full dynamic band structure (which reflects the macroscopic order parameter), we can map and expand the phase diagram using light as a tuning parameter to uncover new understandings of phase transitions as well as new hidden phases in strongly-coupled materials.

Experimentally, trARPES based on HHG is used to measure the dynamic electronic structure, as illustrated in figure 9. In the sudden approximation, the ARPES spectrum can be described as $I(\mathbf{k}, \omega)=I_{0}(\mathbf{k}, \nu, \mathbf{A}) f(\omega) A(\mathbf{k}, \omega)$, where the first term is related to the transition matrix element, while the second and third terms are the temperature dependent Fermi-Dirac function and the spectral function, respectively. By varying the pump-probe delay and the laser fluence, we can systematically map out the band structure, electron temperature, transient heat capacity and the order parameters as a function of time and laser excitation. In the following, we show that this ultrafast electron calorimetry approach offers completely new insights into strongly-coupled materials such as 


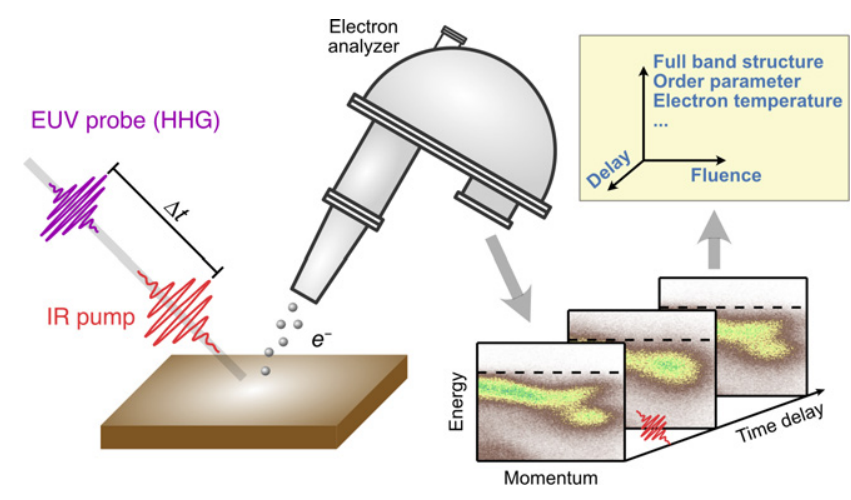

Figure 9. Schematic of the ultrafast electron calorimetry approach based on trARPES. After the material is excited by an ultrashort laser pulse, another EUV pulse from HHG is used to probe the dynamic electronic structure at the controlled time delays. Then the measured band structure, electron temperature and order parameter as a function of both time delay and laser fluence (input energy) can be used to investigate the change in dominant interactions and material state, as well as to map the non-equilibrium phase diagram.

the ultrafast laser-induced magnetic phase transition in $\mathrm{Ni}$, and the charge density wave (CDW) phase transition in $1 T-\mathrm{TaSe}_{2}$.

\subsection{Critical behavior drives the ultrafast magnetic phase transition in nickle}

Since the first experimental observation of ultrafast laserinduced demagnetization [120], this topic has attracted tremendous interest because of its importance to both the fundamental understanding of magnetism as well as for potential applications [121]. However, there has been significant debate about the underlying physical mechanisms and even the relevant timescales. In most past work, laser-induced demagnetization was described as a sequence of events where the energy of the hot electron bath first transfers to the spin and later to the lattice degrees of freedom. This cascade of energy relaxation processes was used to explain why ultrafast demagnetization occurs over a range of timescales from $\sim 100$ to 500 fs. These multiple timescales observed in past experiments, all conducted at many different laser excitation fluences, obscured any contributions from critical phenomena.

Fortunately, in recent work, by implementing HHGbased trARPES and time-resolved magnetooptical Kerr effect (trMOKE) measurements on the same laser-excited sample, we show that critical behavior within 20 fs governs the ultrafast magnetic phase transition in Ni. As explained above, the laser-excited electrons in Ni thermalize very rapidly via electron-electron scattering, reaching a maximum within $\sim 20$ fs. In figure 10(a) we plot the peak electron temperature $\left(T_{\mathrm{e}}\right)$ as a function of laser fluence. Instead of a monotonic increase in temperature, as might be expected if the laser energy is only being absorbed by the electron system (blue dotted line in figure 10(a)), there is a strong suppression as the electron temperature approaches the Curie temperature $T_{\mathrm{C}}$, which occurs at a critical fluence $F_{\text {c }}$. This is consistent with the critical behavior observed during equilibrium magnetic phase transitions - that ferromagnetic magnetic order is lost above the Curie temperature $T_{\mathrm{C}}$. Indeed, the solid red line in figure 10(a) can match our data by assuming that the full electronic heat capacity has contributions both from charges and spins, with a divergence near the Curie temperature $T_{\mathrm{C}}$ (inset of figure 10(a)). This result suggests that the spin system absorbs sufficient energy within 20 fs to subsequently proceed through the magnetic phase transition $[35,36]$.

When comparing the relative magnetization (magnetic asymmetry) measured by trMOKE and the exchange splitting measured by trARPES, we can consistently map the order parameter as a function of time delay and laser fluence, as shown in figure 10(b). First, we found that the demagnetization and recovery dynamics can be described by three universal timescales over the full range of laser fluences for both sets of measurements. Second, although the spin system absorbs sufficient energy within 20 fs to proceed through the phase transition, the demagnetization (indicated by a reduced exchange splitting and magnetic asymmetry) occurs on a much longer timescale of $\sim 176 \mathrm{fs}$. This large time lag between laser energy absorption by the spin system and the appearance of a spectroscopic signature indicates the existence and importance of a transient highly-excited magnetic state during laser-induced demagnetization. Third, remagnetization occurs on timescales of $\sim 537 \mathrm{fs}$ when the laser fluence is below $F_{\mathrm{c}}$ (as shown in figure 10(b)), while an additional slow recovery on a timescale of $\sim 76 \mathrm{ps}$ turns on when the transient $T_{\mathrm{e}}$ exceeds $T_{\mathrm{C}}$. These results define a new timescale of $20 \mathrm{fs}$ in the ultrafast magnetic phase transition, highlight the importance of critical phenomena, and suggest that the transient electron temperature alone dictates the magnetic response $[35,36]$. More recently, we also demonstrated that direct light-induced spin transfer can also occur between two magnetic sublattices, on similarly fast timescales $[37,122]$. In combination, these findings suggest that spin polarization can be manipulated by light on femtosecond and even attosecond timescales.

\subsection{New CDW metastable states in $1 \mathrm{~T}-\mathrm{TaSe}_{2}$ : mode-selective electron-phonon coupling}

We can also use ultrafast electron calorimetry to uncover new phases in CDW materials, as well as to elucidate their origins and to manipulate electron-phonon interactions. At temperatures below $T_{\mathrm{c}} \sim 470 \mathrm{~K}$, the prototypical quasi-2D CDW material $1 T-\mathrm{TaSe}_{2}$ crystallizes into a crystal structure characterized by an electron density modulation and corresponding lattice reconstruction with a $\sqrt{13} \times \sqrt{13}$ star-of-David supercell [123]. Note that in equilibrium, this is a first-order phase transition that is governed by complex electron-phonon interactions. When an ultrashort light pulse excites these materials, the first step is similar to the case for metals above: the laser pulse excites electrons initially, which thermalize rapidly due to strong electron-electron scattering. However, the subsequent relaxation steps are distinct in CDW materials: in $1 T$ $\mathrm{TaSe}_{2}$, the laser-excited electron temperature can reach many thousands of Kelvin, which smears out the charge density since the unoccupied orbitals are spatially delocalized. This fast charge displacement subsequently launches a coherent amplitude (breathing) mode [38] — then either the system will relax 


\section{Electron temperature in $\mathrm{Ni}, 20$ fs into ultrafast magnetic phase transition}

(a)

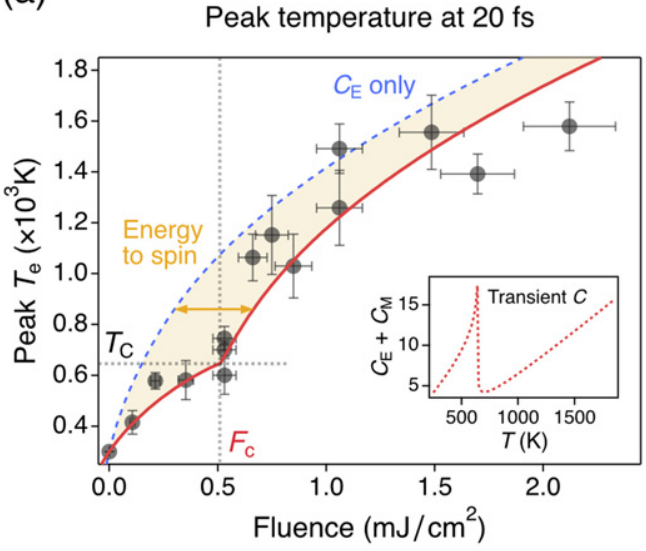

\section{Electron temperature in $1 \mathrm{~T}-\mathrm{TaSe}_{2}$, 4 ps after laser pulse}

(c)

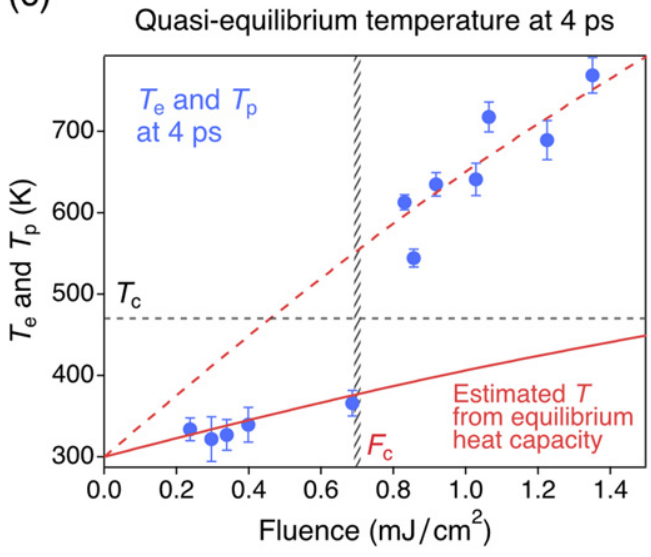

(b)

\section{Order parameter in $\mathrm{Ni}$ - mapping ultrafast magnetic phase transition}

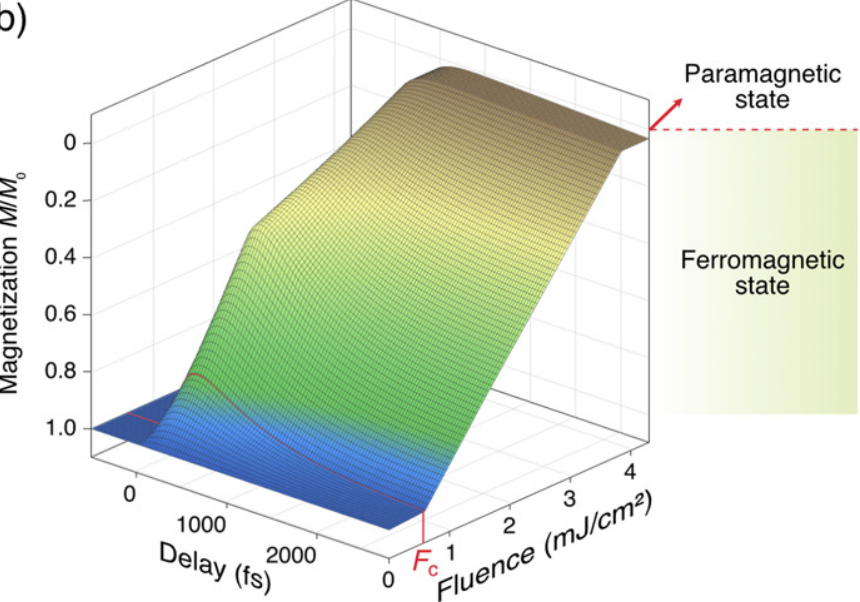

\section{Order parameter in $1 \mathrm{~T}-\mathrm{TaSe}_{2}$ - mapping normal and metastable CDW phases}

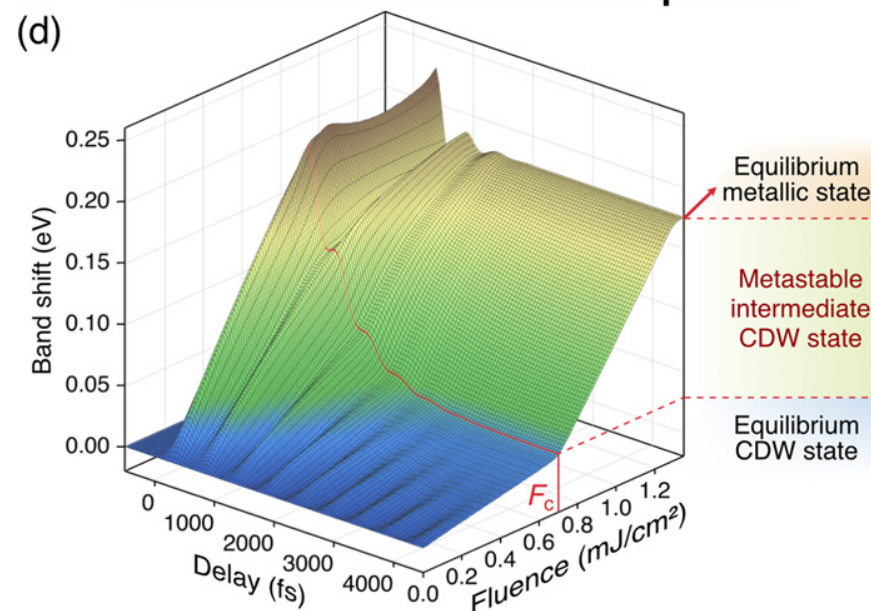

Figure 10. Ultrafast electron calorimetry uncovers new understanding of phase transitions, expanding the phase diagram using light. (a) and (b) are the maps of electron temperature and order parameter for the magnetic phase transition in $\mathrm{Ni}$, respectively. At a critical fluence $F_{\mathrm{c}}$, the transient heat capacity at $20 \mathrm{fs}$ diverges. Such critical behavior governs the subsequent magnetic responses. (c) and (d) are the maps of electron temperature and order parameter for the CDW phase transition in $1 T-\mathrm{TaSe}_{2}$, respectively. Above a critical fluence $F_{\mathrm{c}}$, the effective heat capacity drops by $70 \%$ and the material evolves into a metastable intermediate CDW state. Panel (a) is reproduced from [36]. CC BY 4.0. Panels (c) and (d) are from [38]. Reprinted with permission from AAAS.

back to the ground state or, for sufficiently high laser fluence, the CDW order will melt. This has been known for several years [101, 103, 124].

Using ultrafast electron calorimetry, we found that the phase diagram in $1 T-\mathrm{TaSe}_{2}$ is considerably richer than previously realized. By monitoring the electron temperature as a function of laser fluence, we uncovered a new metastable CDW state in $1 T-\mathrm{TaSe}_{2}$, we found that it is possible to manipulate the electron-phonon couplings by varying the laser excitation, and we observed that the electron temperature is significantly modulated by the coherent amplitude mode [38, 39]. Here we first focus on the relaxation of the hot electrons, which recover to a warm plateau at several picoseconds, when the electron system equilibrates with at least a part of the phonon system. Figure 10(c) displays this quasi-equilibrium temperature as a function of laser fluence. At low fluences, this temperature follows the expected behavior based on the equilibrium heat capacity (the solid red curve). As the laser fluence is increased beyond a critical value $F_{\mathrm{c}}$, there is an abrupt jump in both the temperature and the slope-indicating a reduction in the effective heat capacity of the system by as much as $70 \%$. Since the heat capacity cannot change so radically under equilibrium conditions, these results suggest that only a subset of phonons are strongly coupled to the hot electrons at fluences higher than $F_{\mathrm{c}}[38,39]$. Physically, in an overly simplified picture, one might expect that ultrafast laser excitation is a unique way of isolating the interactions between the charge and the strongly coupled phonons (often in-plane), 
since weakly-coupled phonons (often cross-plane) will not be excited until several tens of picoseconds. Thus, ultrafast laser excitation may modify the interlayer coupling and/or stacking, e.g., transform a material from quasi-2D to more 2D, thereby making it possible to support new phases. This also implies that there could be dramatic variations in the electron-phonon interactions, which we observe.

In order to see how anomalous electron-phonon coupling can change the state of the material, we use the band shift as an order parameter to map the non-equilibrium phase diagram, as shown in figure 10(d) [38]. At the same critical fluence $F_{\mathrm{c}}$, the CDW order is completely melted initially, while the quasi-equilibrium order at several picoseconds shows a kink. In other words, when the electron-phonon coupling becomes mode-selective at high fluences, the inhomogeneity within the phonon system drives the material into a new metastable, intermediate, CDW state, which is distinct from all the known equilibrium states. Thus, ultrafast laser excitation can enrich the phase diagram of $1 T-\mathrm{TaSe}_{2}$. Most importantly, because of the substantially reduced effective heat capacity of the metastable state, this transformation is more energy efficient than under equilibrium conditions.

In addition to observing a new intermediate CDW state with very interesting properties, ultrafast electron calorimetry can reveal many more intriguing phenomena. As an example, we also observe a large coherent modulation of the electron temperature, that is coupled to the amplitude mode, but exhibits a phase shift of $\pi$ at the critical fluence [39]. This finding is in contrast to all previous observations in multiple materials to date, where the electron temperature decreases monotonically via electron-phonon scattering [125]. By tuning the laser fluence, the magnitude of the electron temperature modulation changes from $\sim 200 \mathrm{~K}$ in the case of weak excitation, to $\sim 1000 \mathrm{~K}$ for strong laser excitation. The phase change of $\pi$ in the electron temperature modulation at a critical fluence of $0.7 \mathrm{~mJ} \mathrm{~cm}^{-2}$ suggests a switching of the dominant coupling mechanism between the coherent phonon and electrons [39]. Ultrafast electron calorimetry therefore opens up routes for coherently manipulating the interactions and properties of $2 \mathrm{D}$ and other quantum materials using light.

\subsection{Summary}

In summary, ultrafast electron calorimetry combines ultrafast laser excitation with HHG-based trARPES to provide a new and powerful technique to capture and control microscopic interactions, novel excited and metastable states, and phase transitions. It can map how ultrafast laser excitation expands the phase diagram of materials to uncover many new phenomena that cannot be accessed through equilibrium heating. The new findings include how critical behavior and a highly excited magnetic state drives the ultrafast magnetic phase transition in $\mathrm{Ni}$, as well as a new metastable CDW state in the quasi $2 \mathrm{D}$ material $1 T-\mathrm{TaSe}_{2}$ that is mediated by mode-selective electron-phonon coupling. We note that this approach demonstrates a novel way to manipulate phase transitions and electron-phonon interactions, opening up new routes to steer quantum materials toward the desired state using light.

\section{Sub-wavelength short wavelength imaging using coherent HHG beams}

\subsection{Introduction}

In addition to enabling attosecond spectroscopy, HHG has also recently attracted attention for applications in coherent imaging and dynamic scatterometry [40-42, 92, 126-128]. The theoretical resolution of any imaging system is limited by the diffraction of light. For example, the Abbe diffraction limit sets the transverse spatial resolution at $\sim \lambda /(2 \mathrm{NA})$, where NA represents the numerical aperture of the imaging system. Thus, the shorter wavelength of HHG EUV or X-ray sources could theoretically outperform visible or UV microscopy by 2-3 orders of magnitude - with the additional advantages of elemental/chemical/magnetic contrast, high time resolution, and the ability to penetrate layers that are opaque to visible light. However, practical limits due to imperfections in the imaging optics, aberrations, or misalignment impede the performance of traditional approaches. As a result, conventional xray microscopes using zone plates as focusing and imaging optics can only achieve $\sim 10 x$ the diffraction limit [129].

Fortunately, coherent HHG beams are ideal illumination sources for coherent diffraction imaging (CDI) and scatterometry techniques. CDI is a lensless computational imaging method that replaces EUV/x-ray imaging optics with an iterative phase retrieval algorithm that recovers the real-space image of the sample from the measured diffraction patterns in reciprocal (Fourier) space, as illustrated in figure 11 [130, 131]. Initial implementations of CDI used a simple illumination geometry and recovered the image of the sample by averaging over $\sim 200$ different reconstructions.

More recently, a powerful new form of CDI called ptychography [132] has emerged as a high-fidelity CDI method that enables complex image reconstruction of an extended sample with a high spatial resolution. In ptychography, an illumination beam (probe) scans a sample (object) at multiple partially overlapped locations generating a set of diffraction patterns. In this scanning geometry, the information redundancy from the overlapping diffraction patterns can be exploited to achieve robust computational image reconstruction. The reconstruction pixel size, $d_{x}$, is given by $d_{x}=\lambda * z /\left(N * p_{x}\right)$, where $\lambda$ is the wavelength, $z$ is the distance from an image detector (such as an x-ray camera) to the sample, $N$ is the number of pixels on the detector and $p_{x}$ is the effective pixel size. Therefore, the resolution performance in ptychographic CDI is no longer limited by the manufacturing quality of optics, enabling high resolution imaging near the diffraction limit. Modern ptychographic algorithms also make it possible to reconstruct complex (amplitude and phase) images of both the beam and the sample simultaneously.

\subsection{Record sub-wavelength short wavelength imaging}

Using ptychographic CDI with tabletop HHG EUV centered at $13.5 \mathrm{~nm}$, a recent experiment has demonstrated the first sub-wavelength EUV image using any light source - tabletop or facility scale. This work essentially demonstrated the first near-perfect EUV/SXR microscope. We achieved full field, 


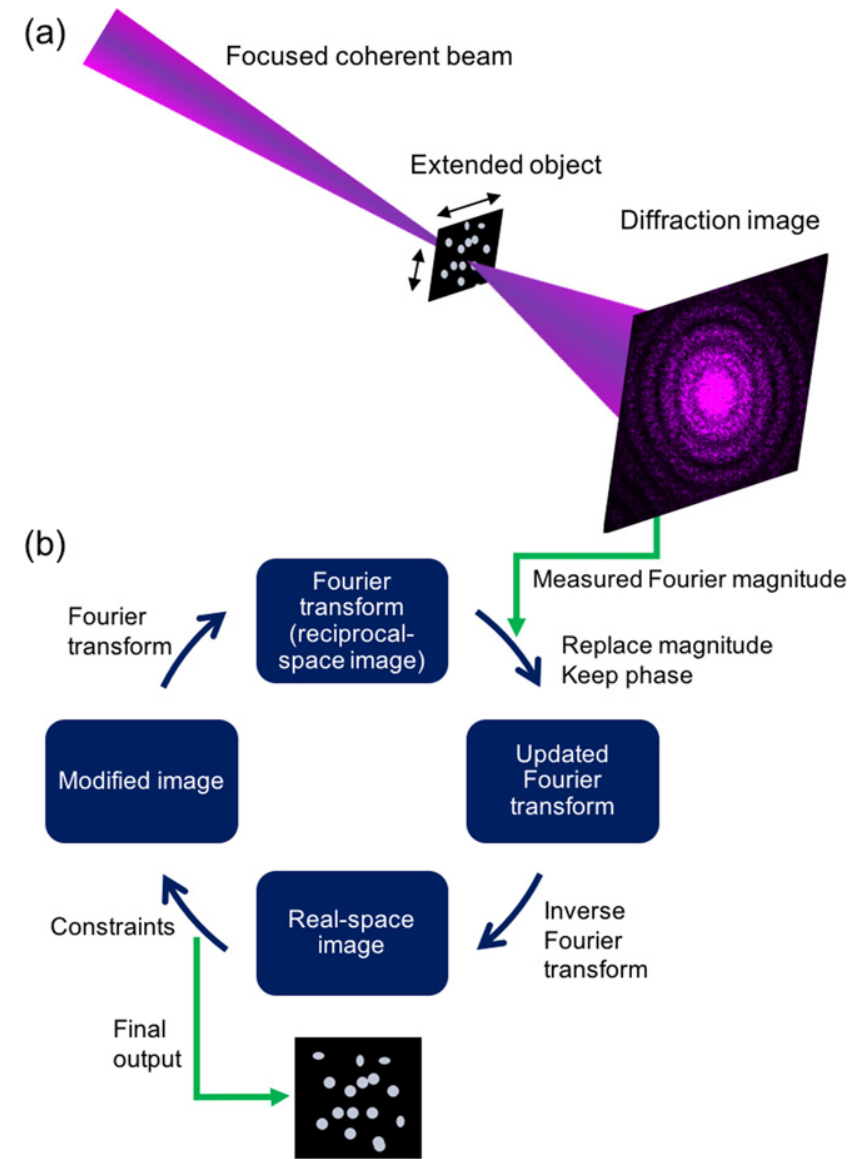

Figure 11. Schematic of the CDI method using iterative phase retrieval algorithms. (a) In ptychographic CDI, a coherent beam (probe) is focused on an extended sample (object), which is scanned through the probe on a 2D grid with partial overlaps. (b) A phase retrieval algorithm then iterates back and forth between real- and reciprocal- (Fourier) space. Various constraints and supports are enforced in real-space at each iteration until the errors are small and the correct phase is recovered as the final output complex image.

large area, quantitative, transmission-mode imaging of a nearperiodic sample with a transverse spatial resolution of $0.9 \lambda$, or $12.6 \mathrm{~nm}$ at a wavelength of $13.5 \mathrm{~nm}$, as shown in figure 12 [40]. This work combined a new method called modulus enforced probe (MEP), which is based on collecting a single image of the unscattered direct beam and applying it as a constraint on the probe within a novel algorithm based on the extended ptychographic iterative engine algorithm (ePIE). Therefore, an extended, nearly periodic sample can be imaged with a highresolution and extremely high-fidelity for the first time. Using large-scale synchrotron sources, a spatial resolution of a few nanometers $(\sim 2 \mathrm{~nm}$ in $2 \mathrm{D}$ and $\sim 5.5 \mathrm{~nm}$ in $3 \mathrm{D})$ was achieved using sub-2 $\mathrm{nm}$ wavelength $\mathrm{x}$-rays [133-136].

Finally, it is worth mentioning that the concept of ptychography can also be extended into time domain for attosecond pulse reconstruction [137] as a method to characterize highly broad bandwidth HHG sources. This approach complements other techniques such as attosecond streaking and ARPES. The latter was used to fully characterize more complex circularly-polarized attosecond waveforms, by harnessing the strong polarization selectivity in photoemission from solid surfaces, as well as the ability to rotate the circularly polarized
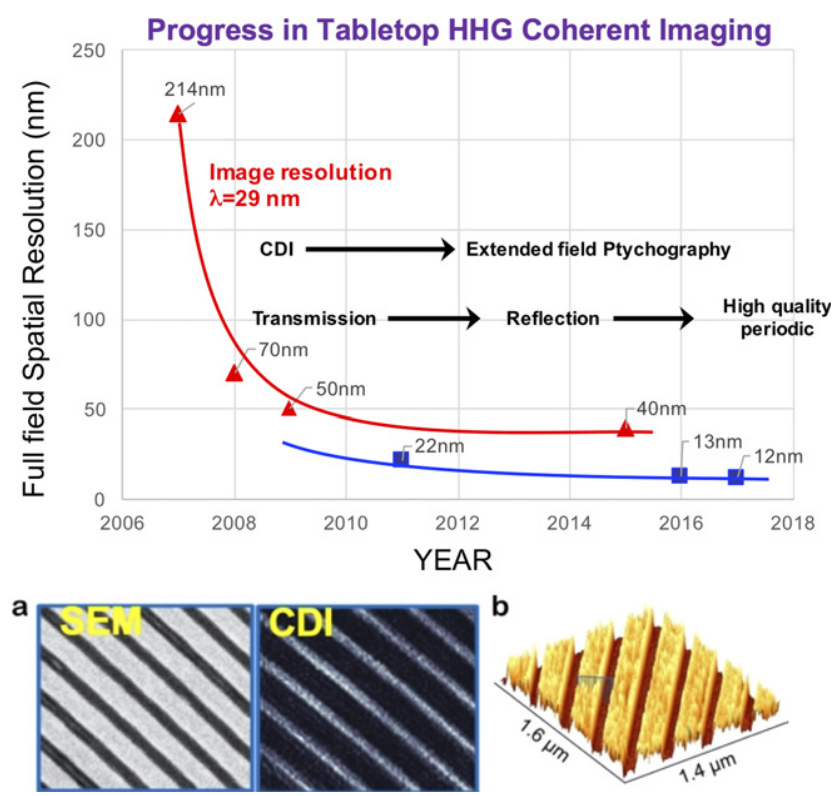

Figure 12. (top) Progress in full field tabletop transverse spatial resolution enabled by HHG. Red: image resolution using EUV at a wavelength of $29 \mathrm{~nm}$. Blue: image resolution using EUV at a wavelength of $13.5 \mathrm{~nm}$. (bottom) 1st sub-wavelength short wavelength imaging, achieving a spatial resolution of $12.6 \mathrm{~nm}$ at a wavelength of $13.5 \mathrm{~nm}$. (a) SEM scanning electron microscope and CDI images. (b) Height map of the sample. The bottom panel is reprinted by permission from Springer Nature Customer Service Centre GmbH: Springer Nature, Nature Photonics [40] 2017.

EUV field in space. This makes it possible to fully characterize some of the most complex coherent light fields to date, where the spectral, temporal and polarization states are rapidly changing on attosecond timescales [27].

\subsection{Dynamic imaging and scatterometry using HHG beams}

Static and dynamic ptychographic full field imaging or scatterometry can also be implemented in reflection geometries to image buried layers and interfaces [41], or to capture spin, charge, acoustic and heat transport [92, 126, 138]. The short wavelength and high time resolution of HHG sources provides enhanced sensitivity to buried interfaces. Because the depth resolution of these buried interfaces is a result of the phase, we can achieve a deep sub- $\AA$ depth resolution, making it possible to address important nanoscale transport challenges. For example, at dimensions $<100 \mathrm{~nm}$, bulk models no longer accurately predict the transport, mechanical, or phase properties of materials, and visible probes have limited sensitivity. Dynamic EUV scatterometry can better capture nanoscale heat flow to validate and generalize a surprising prediction - an array of closely-spaced nanoscale heat sources can cool more quickly than when spaced far apart due to better coupling to the phonon spectrum in the substrate $[92,138]$. This is leading to better predictive theories that may guide the design of energyefficient nano-devices, where better thermal management is critically needed.

Combing ptychography with ultrafast pump-probe techniques makes it possible to create nanoscale real-time movies with $<10$ fs time resolution, and in principle with attosecond time resolution. For example, the first full-field 


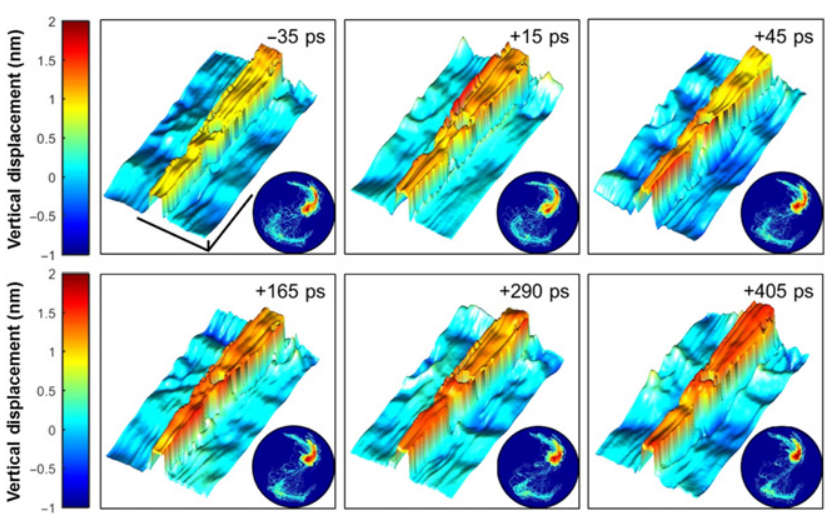

Figure 13. Nanoscale imaging enabled by HHG. The data represents the first full field dynamic movie of acoustic waves, with $\sim 10 \mathrm{fs}$ temporal resolution. The scale bars indicated are $5 \mu \mathrm{m} \times 5 \mu \mathrm{m}$ $\times 0.5 \mathrm{~nm}$. Reproduced from [143]. CC BY 4.0 .

HHG ptychographic movie imaged thermal and acoustic dynamics in a nanoantenna with sub-100 nm transverse and 0.5 Å depth spatial resolution, combined with $\sim 10$ fs temporal resolution [126]. As shown in figure 13, this experiment directly visualized the complex thermal and acoustic response of an individual nanoscale antenna on a substrate after impulsive heating by a femtosecond laser.

For dynamic imaging, it would also be useful to use coherent broadband EUV or SXR HHG sources to image spin and charge transport, for example. However, implementing broadband ptychography is very challenging in terms of its phaseretrieval algorithms, which does not favor broadband illumination. The recent development and refinement of new ptychographic algorithms suggest new phase-retrieval algorithms that could lead to the future implementation of attosecond-resolved nanoscale ptychographic imaging $[139,140]$.

Another important aspect of EUV and x-ray ptychographic imaging is the ability to extract the chemical composition of the sample. Quantitative composition extraction can be accomplished by angle-scanning in reflection mode ptychography to implement complex imaging reflectometry [127], or by wavelength-scanning in transmission mode ptychography $[141,142]$.

\subsection{Summary}

High harmonic based coherent imaging and scattering has already achieved the first sub-wavelength resolution full field imaging using any EUV or x-ray light source, uncovered new regimes of nanoscale thermal transport, and enabled nondestructive characterization of the structure and properties of heterostructures. In the future, it may be possible to extend super-resolution imaging to shorter wavelengths via OAM beams, to enable fast imaging of multilayer and chiral structures being investigated for future quantum, energy, electronic and storage devices.

\section{Conclusion}

Three decades of study of the attosecond dynamics of HHG, and the applications of these sources for time-resolved studies, have demonstrated just how vital this research area is, as well as the extraordinary potential for further advances. Even as we learn more about the HHG process itself, its utility as a coherent light source in a new spectral region is propelling a rapid expansion in the sphere of influence of this area. Like in many fields, exploration of new regimes yields both esoteric findings of interest to experts, as well as fundamentally new light science technologies that are impacting other fields. Other schemes that are also being explored for attosecond pulse generation include nonlinear Thomson backscattering on relativistic electron bunches, relativistic oscillating mirrors, or laser-perturbed or seeded x-ray free-electron lasers (XFELs).

The continued development of ultrafast laser and X-ray technologies will continue to enable new physics and technology applications. Looking forward, important scientific challenges to be addressed include the photon energy limits of phase matching of high harmonic generation, which can likely extend into the $>10 \mathrm{keV}$, hard $\mathrm{x}$-ray, region. Addressing this question will require new mid-IR ultrafast lasers, as well as advanced computing capabilities that can combine multi-color electromagnetic propagation (Maxwell's equations) with the 3D time-dependent Schrödinger equation. Finally, many important science and technological challenges can be addressed by the extension of magnetic spectroscopies and imaging to the deeper bound $L$-edge region, where the spin and orbital contributions to the magnetic properties can be separated.

\section{Acknowledgments}

We gratefully acknowledge support from the National Science Foundation through the JILA Physics Frontiers Center PHY-1734006, and a Gordon and Betty Moore Foundation EPiQS Award GBMF4538, for the attosecond ARPES setups and methods. We gratefully acknowledge support from the Department of Energy Office of Basic Energy Sciences XRay Scattering Program Award DE-SC0002002 for the spin dynamics setups and methods. We gratefully acknowledge support from the Department of Energy Office of Basic Energy Sciences AMOS Award DE-FG02-99ER14982 (experiment) as well as an MURI Grant from the Air Force Office of Scientific Research under Award Number FA9550-16-1-0121 (theory) for the HHG light source setups and methods. The authors gratefully acknowledge support from the STROBE National Science Foundation Science and Technology Center, Grant No. DMR-1548924, for the imaging setups and methods. C H-G acknowledges support by Ministerio de Ciencia, Innovación y Universidades for projects (FIS2016-75652P and PID2019-106910GB-I00) and a Ramón y Cajal contract (RYC-2017-22745), co-funded by the European Social Fund; Junta de Castilla y León and European Union, FEDER, (Project SA287P18); and from the European Research Council (ERC) under the European Union's Horizon 2020 research and innovation program (Grant agreement No. 851201). Z $\mathrm{T}$ gratefully acknowledges the financial support from the National Natural Science Foundation of China (Grant No. 11874121), the Shanghai Municipal Science and Technology Basic Research Project (Grant No. 19JC1410900) and the Alexander-von-Humboldt foundation. 


\section{ORCID iDs}

Xun Shi (D) https://orcid.org/0000-0001-8719-911X

Chen-Ting Liao (i) https://orcid.org/0000-0002-3423-277X

Emma Cating-Subramanian (D) https://orcid.org/0000-00016040-5622

Carlos Hernández-García (D) https://orcid.org/0000-00026153-2647

\section{References}

[1] McPherson A et al 1987 Studies of multiphoton production of vacuum-ultraviolet radiation in the rare gasses J. Opt. Soc. Am. B 4 595-601

[2] Ferray M et al 1988 Multiple-harmonic conversion of 1064 Nm radiation in rare-gases J. Phys. B: At. Mol. Opt. Phys. 21 L31-5

[3] Kuchiev M Y 1987 Atomic antenna JETP Lett. 45 404-6

[4] Farkas G and Tóth C 1992 Proposal for attosecond light pulse generation using laser induced multiple-harmonic conversion processes in rare gases Phys. Lett. A $168447-50$

[5] Kulander K C and Shore B W 1989 Calculations of multipleharmonic conversion of 1064 NM radiation in Xe Phys. Rev. Lett. 62 524-6

[6] Zhou J, Huang C P, Shi C, Murnane M M and Kapteyn H C 1994 Generation of $21 \mathrm{fs}$ millijoule-energy pulses by use of Ti:sapphire Opt. Lett. 19 126-8

[7] Asaki M T et al 1993 Generation of 11 fs pulses from a modelocked Ti:sapphire laser Opt. Lett. 18977

[8] Backus S, Durfee C, Murnane M M and Kapteyn H C 1998 High power ultrafast lasers Rev. Sci. Instrum. 69 1207-23

[9] Zhou J, Peatross J, Murnane M M, Kapteyn H C and Christov I P 1996 Enhanced high harmonic generation using 25 femtosecond laser pulses Phys. Rev. Lett. 76 752-5

[10] Chang Z et al 1998 Temporal phase control of soft-x-ray harmonic emission Phys. Rev. A 58 R30-3

[11] Chang Z, Rundquist A, Wang H, Murnane M and Kapteyn H 1997 Generation of coherent soft $x$ rays at $2.7 \mathrm{~nm}$ using high harmonics Phys. Rev. Lett. 79 2967-70

[12] Lewenstein M, Balcou P, Ivanov M Y, L'Huillier A and Corkum P B 1994 Theory of high-harmonic generation by low-frequency laser fields Phys. Rev. A 49 2117-32

[13] Bartels R et al 2000 Shaped-pulse optimization of coherent emission of high-harmonic soft x-rays Nature 406 164-6

[14] Rundquist A et al 1998 Phase-matched generation of coherent soft x-rays Science 280 1412-5

[15] Bartels R A et al 2002 Generation of spatially coherent light at extreme ultraviolet wavelengths Science 297 376-8

[16] Bartels R, Backus S, Christov I, Kapteyn H and Murnane M 2001 Attosecond time-scale feedback control of coherent $\mathrm{x}$ ray generation Chem. Phys. 267 277-89

[17] Christov I, Murnane M and Kapteyn H 1997 High-harmonic generation of attosecond pulses in the 'single-cycle' regime Phys. Rev. Lett. 78 1251-4

[18] Baltuska A et al 2003 Attosecond control of electronic processes by intense light fields Nature $\mathbf{4 2 1} 611-5$

[19] Nisoli M, De Silvestri S and Svelto O 1996 Generation of highenergy 10 fs pulses by a new pulse compression technique Appl. Phys. Lett. 68 2793-5

[20] Christov I, Murnane M and Kapteyn H 1998 Generation and propagation of attosecond $\mathrm{x}$-ray pulses in gaseous media Phys. Rev. A 57 R2285-8

[21] Popmintchev T et al 2009 Phase matching of high harmonic generation in the soft and hard $\mathrm{x}$-ray regions of the spectrum Proc. Natl Acad. Sci. USA 106 10516-21
[22] Popmintchev T et al 2012 Bright coherent ultrahigh harmonics in the keV x-ray regime from mid-infrared femtosecond lasers Science 336 1287-91

[23] Popmintchev T et al 2008 Extended phase matching of high harmonics driven by mid-infrared light Opt. Lett. 33 2128-30

[24] Kfir O et al 2015 Generation of bright phase-matched circularly-polarized extreme ultraviolet high harmonics Nat. Photon. 9 99-105

[25] Fan T et al 2015 Bright circularly polarized soft x-ray high harmonics for x-ray magnetic circular dichroism Proc. Natl Acad. Sci. USA 112 14206-11

[26] Hernandez-Garcia C et al 2016 Schemes for generation of isolated attosecond pulses of pure circular polarization Phys. Rev. A 93043855

[27] Chen C et al 2016 Tomographic reconstruction of circularly polarized high-harmonic fields: 3D attosecond metrology Sci. Adv. 28

[28] Huang P-C et al 2018 Polarization control of isolated highharmonic pulses Nat. Photon. 12 349-54

[29] Dorney K et al 2017 Helicity-selective enhancement and polarization control of attosecond high harmonic waveforms driven by bichromatic circularly polarized laser fields $P$ hys. Rev. Lett. 119063201

[30] Hickstein D D et al 2015 Non-collinear generation of angularly isolated circularly polarized high harmonics Nat. Photon. 9 743

[31] Rego L et al 2019 Generation of extreme-ultraviolet beams with time-varying orbital angular momentum Science $\mathbf{3 6 4}$ eaaw 9486

[32] Dorney K M et al 2019 Controlling the polarization and vortex charge of attosecond high-harmonic beams via simultaneous spin-orbit momentum conservation Nat. Photon. 13 $123-30$

[33] Tao Z S et al 2016 Direct time-domain observation of attosecond final-state lifetimes in photoemission from solids $\mathrm{Sci}$ ence 353 62-7

[34] Chen C et al 2017 Distinguishing attosecond electron-electron scattering and screening in transition metals Proc. Natl Acad. Sci. 114 E5300-7

[35] You W et al 2018 Revealing the universal nature of the ultrafast magnetic phase transition in Ni by correlating extreme ultraviolet magneto-optic and photoemission spectroscopies Phys. Rev. Lett. 121077204

[36] Tengdin P et al 2018 Critical behavior within 20 fs drives the out-of-equilibrium laser-induced magnetic phase transition in nickel Sci. Adv. 49744

[37] Tengdin P et al 2020 Direct light-induced spin transfer between different elements in a spintronic Heusler material via femtosecond laser excitation Sci. Adv. 67

[38] Shi X et al 2019 Ultrafast electron calorimetry uncovers a new long-lived metastable state in $1 \mathrm{~T}-\mathrm{TaSe}_{2}$ mediated by modeselective electron-phonon coupling $S c i$. Adv. 5 eaav4449

[39] Zhang Y et al 2020 Coherent modulation of the electron temperature and electron-phonon couplings in a 2D material Proc. Natl Acad. Sci. USA 117 8788-93

[40] Gardner D F et al 2017 Sub-wavelength coherent imaging of periodic samples using a $13.5 \mathrm{~nm}$ tabletop high-harmonic light source Nat. Photon. 11 259-63

[41] Shanblatt E R et al 2016 Quantitative chemically-specific coherent diffractive imaging of reactions at buried interfaces with few-nanometer precision Nano Lett. 16 5444-50

[42] Miao J, Ishikawa T, Robinson I K and Murnane M M 2015 Beyond crystallography: diffractive imaging using coherent $\mathrm{x}$-ray light sources Science 348 530-5

[43] Hernandez-Garcia C et al 2013 Zeptosecond high harmonic $\mathrm{keV} \mathrm{x}$-ray waveforms driven by midinfrared laser pulses Phys. Rev. Lett. 111033002 
[44] Popmintchev T, Chen M C, Arpin P, Murnane M M and Kapteyn H C 2010 The attosecond nonlinear optics of bright coherent x-ray generation Nat. Photon. 4 822-32

[45] Chen M C et al 2010 Bright, coherent, ultrafast soft x-ray harmonics spanning the water window from a tabletop light source Phys. Rev. Lett. 105173901

[46] Galloway B R et al 2016 Lorentz drift compensation in high harmonic generation in the soft and hard x-ray regions of the spectrum Opt. Express $2421818-32$

[47] Hernández-García C et al 2016 Group velocity matching in high-order harmonic generation driven by mid-infrared lasers New J. Phys. 18073031

[48] Hernández-García C et al 2017 Isolated broadband attosecond pulse generation with near- and mid-infrared driver pulses via time-gated phase matching Opt. Express $\mathbf{2 5}$ $11855-66$

[49] Schoenlein R et al 2019 Recent advances in ultrafast x-ray sources Phil. Trans. R. Soc. A 37720180384

[50] Ellis J L et al 2018 High harmonics with spatially varying ellipticity Optica 5 479-85

[51] Dorney K M et al 2019 Controlling the polarization and vortex charge of attosecond high-harmonic beams via simultaneous spin-orbit momentum conservation Nat. Photon. 13 123

[52] Allen L, Beijersbergen M W, Spreeuw R J C and Woerdman J P 1992 Orbital angular momentum of light and the transformation of Laguerre-Gaussian laser modes Phys. Rev. A 45 8185-9

[53] Vodungbo B et al 2011 Polarization control of high order harmonics in the EUV photon energy range Opt. Express $\mathbf{1 9}$ 4346-56

[54] Dietrich P, Burnett N H, Ivanov M and Corkum P B 1994 Highharmonic generation and correlated two-electron multiphoton ionization with elliptically polarized light Phys. Rev. A 50 R3585-8

[55] Becker W, Chichkov B N and Wellegehausen B 1999 Schemes for the generation of circularly polarized highorder harmonics by two-color mixing Phys. Rev. A 60 1721-2

[56] Eichmann H et al 1995 Polarization-dependent high-order twocolor mixing Phys. Rev. A 51 R3414-7

[57] Azoury D et al 2019 Interferometric attosecond lock-in measurement of extreme-ultraviolet circular dichroism Nat. Photon. 13 198-204

[58] Hernández-García C et al 2017 Extreme ultraviolet vector beams driven by infrared lasers Optica 4 520-6

[59] Turpin A, Rego L, Picón A, San Román J and HernándezGarcía C 2017 Extreme ultraviolet fractional orbital angular momentum beams from high harmonic generation Sci. Rep. 743888

[60] Hernández-García C et al 2017 Generation and applications of extreme-ultraviolet vortices Photonics 428

[61] Hernández-García C 2017 A twist in coherent x-rays Nat. Phys. 13 327-9

[62] Zürch M, Kern C, Hansinger P, Dreischuh A and Spielmann C 2012 Strong-field physics with singular light beams Nat. Phys. 8 743-6

[63] Hernández-García C, Picón A, San Román J and Plaja L 2013 Attosecond extreme ultraviolet vortices from high-order harmonic generation Phys. Rev. Lett. 11183602

[64] Géneaux R et al 2016 Synthesis and characterization of attosecond light vortices in the extreme ultraviolet Nat. Commun. 712583

[65] Kong F et al 2017 Controlling the orbital angular momentum of high harmonic vortices Nat. Commun. 814970

[66] Gauthier D et al 2017 Tunable orbital angular momentum in high-harmonic generation Nat. Commun. 814971

[67] Rego L, Román J S, Picón A, Plaja L and HernándezGarcía C 2016 Nonperturbative twist in the generation of extreme-ultraviolet vortex beams Phys. Rev. Lett. 117 163202

[68] Fujita H and Sato M 2017 Ultrafast generation of skyrmionic defects with vortex beams: printing laser profiles on magnets Phys. Rev. B 95054421

[69] Sirenko A A et al 2019 Terahertz vortex beam as a spectroscopic probe of magnetic excitations Phys. Rev. Lett. 122 237401

[70] van Veenendaal M 2015 Interaction between x-ray and magnetic vortices Phys. Rev. B 92245116

[71] Cavalieri A L et al 2007 Attosecond spectroscopy in condensed matter Nature 449 1029-32

[72] Neppl S et al 2012 Attosecond time-resolved photoemission from core and valence states of magnesium Phys. Rev. Lett. 109087401

[73] Miaja-Avila L et al 2008 Direct measurement of core-level relaxation dynamics on a surface-adsorbate system Phys. Rev. Lett. 101046101

[74] Dahlström J M et al 2013 Theory of attosecond delays in laserassisted photoionization Chem. Phys. 414 53-64

[75] Neppl S et al 2015 Direct observation of electron propagation and dielectric screening on the atomic length scale Nature $517342-6$

[76] Locher R et al 2015 Energy-dependent photoemission delays from noble metal surfaces by attosecond interferometry Optica 2 405-10

[77] Siek F et al 2017 Angular momentum-induced delays in solidstate photoemission enhanced by intra-atomic interactions Science 357 1274-7

[78] Miaja-Avila L et al 2006 Laser-assisted photoelectric effect from surfaces Phys. Rev. Lett. 97113604

[79] Paul P M et al 2001 Observation of a train of attosecond pulses from high harmonic generation Science $\mathbf{2 9 2}$ 1689-92

[80] Klünder K et al 2011 Probing single-photon ionization on the attosecond time scale Phys. Rev. Lett. 106143002

[81] Hermanson J 1977 Final-state symmetry and polarization effects in angle-resolved photoemission spectroscopy Solid State Commun. 22 9-11

[82] Himpsel F J, Knapp J A and Eastman D E 1979 Experimental energy-band dispersions and exchange splitting for Ni Phys. Rev. B 19 2919-27

[83] Echenique P M, Pitarke J M, Chulkov E V and Rubio A 2000 Theory of inelastic lifetimes of low-energy electrons in metals Chem. Phys. 251 1-35

[84] Ladstädter F, Hohenester U, Puschnig P and Ambrosch-Draxl C 2004 First-principles calculation of hot-electron scattering in metals Phys. Rev. B 70235125

[85] Campillo I, Pitarke J M, Rubio A, Zarate E and Echenique P M 1999 Inelastic lifetimes of hot electrons in real metals Phys. Rev. Lett. 83 2230-3

[86] Knorren R, Bennemann K H, Burgermeister R and Aeschlimann M 2000 Dynamics of excited electrons in copper and ferromagnetic transition metals: theory and experiment Phys. Rev. B 61 9427-40

[87] Bauer M, Marienfeld A and Aeschlimann M 2015 Hot electron lifetimes in metals probed by time-resolved two-photon photoemission Prog. Surf. Sci. 90 319-76

[88] Grüner G 1988 The dynamics of charge-density waves Rev. Mod. Phys. 60 1129-81

[89] Scalapino D J 2012 A common thread: the pairing interaction for unconventional superconductors Rev. Mod. Phys. $\mathbf{8 4}$ $1383-417$

[90] Giustino F 2017 Electron-phonon interactions from first principles Rev. Mod. Phys. 8915003

[91] Popmintchev D et al 2018 Near and extended edge x-ray absorption fine structure spectroscopy using ultrafast coherent high harmonic supercontinua Phys. Rev. Lett. 120 093002 
[92] Hoogeboom-Pot K M et al 2015 A new regime of nanoscale thermal transport: collective diffusion increases dissipation efficiency Proc. Natl Acad. Sci. 112 4846-51

[93] Turgut E et al 2013 Controlling the competition between optically induced ultrafast spin-flip scattering and spin transport in magnetic multilayers Phys. Rev. Lett. 110197201

[94] Siemens M E et al 2010 Quasi-ballistic thermal transport from nanoscale interfaces observed using ultrafast coherent soft X-ray beams Nat. Mater. 9 26-30

[95] La-O-Vorakiat C et al 2009 Ultrafast demagnetization dynamics at the $\mathrm{M}$ edges of magnetic elements observed using a tabletop high-harmonic soft x-ray source Phys. Rev. Lett. 103257402

[96] Mathias S et al 2007 Angle-resolved photoemission spectroscopy with a femtosecond high harmonic light source using a two-dimensional imaging electron analyzer Rev. Sci. Instrum. 78083105

[97] Bauer M, Lei C, Tobey R, Murnane M M and Kapteyn H 2003 Time-resolved UPS: a new experimental technique for the study of surface chemical reactions on femtosecond timescales Surf. Sci. 532 1159-65

[98] Bauer M et al 2001 Direct observation of surface chemistry using ultrafast soft-x-ray pulses Phys. Rev. Lett. 8702 5501-U5559

[99] Backus S, Peatross J, Huang C P, Kapteyn H C and Murnane M M 1995 Ti:Sapphire amplifier producing millijoule-level, $21 \mathrm{fs}$ pulses at $1 \mathrm{kHz}$ Opt. Lett. 202000

[100] Fritz D M et al 2007 Ultrafast bond softening in bismuth: mapping a solid's interatomic potential with x-rays Science $\mathbf{3 1 5}$ 633-6

[101] Schmitt F et al 2008 Transient electronic structure and melting of a charge density wave in $\mathrm{TbTe}_{3}$ Science 321 1649-52

[102] Dal Conte S et al 2012 Disentangling the electronic and phononic glue in a high-Tc superconductor Science $\mathbf{3 3 5}$ 1600-3

[103] Rohwer $\mathrm{T}$ et al 2011 Collapse of long-range charge order tracked by time-resolved photoemission at high momenta Nature $\mathbf{4 7 1} 490$

[104] Smallwood C L et al 2012 Tracking Cooper pairs in a cuprate superconductor by ultrafast angle-resolved photoemission Science 336 1137-9

[105] Wang Y H, Steinberg H, Jarillo-Herrero P and Gedik N 2013 Observation of Floquet-Bloch states on the surface of a topological insulator Science 342 453-7

[106] Stojchevska L et al 2014 Ultrafast switching to a stable hidden quantum state in an electronic crystal Science $\mathbf{3 4 4}$ $177-80$

[107] Matsunaga R et al 2014 Light-induced collective pseudospin precession resonating with Higgs mode in a superconductor Science 3451145

[108] Gerber S et al 2017 Femtosecond electron-phonon lock-in by photoemission and x-ray free-electron laser Science $\mathbf{3 5 7}$ $71-5$

[109] Harter J W, Zhao Z Y, Yan J Q, Mandrus D G and Hsieh D 2017 A parity-breaking electronic nematic phase transition in the spin-orbit coupled metal $\mathrm{Cd}_{2} \mathrm{Re}_{2} \mathrm{O}_{7}$ Science 356 295

[110] Nicholson C W et al 2018 Beyond the molecular movie: dynamics of bands and bonds during a photoinduced phase transition Science $\mathbf{3 6 2} 821$

[111] Reimann J et al 2018 Subcycle observation of lightwave-driven Dirac currents in a topological surface band Nature $\mathbf{5 6 2}$ 396-400

[112] Na M et al 2019 Direct determination of mode-projected electron-phonon coupling in the time domain Science 366 1231-6

[113] Rini M et al 2007 Control of the electronic phase of a manganite by mode-selective vibrational excitation Nature $\mathbf{4 4 9}$ $72-4$
[114] Fausti D et al 2011 Light-induced superconductivity in a stripeordered cuprate Science 331 189-91

[115] Mankowsky R et al 2014 Nonlinear lattice dynamics as a basis for enhanced superconductivity in $\mathrm{YBa}_{2} \mathrm{Cu}_{3} \mathrm{O}_{6.5}$ Nature $\mathbf{5 1 6}$ 71-3

[116] Mitrano M et al 2016 Possible light-induced superconductivity in $\mathrm{K} 3 \mathrm{C} 60$ at high temperature Nature $\mathbf{5 3 0}$ $461-4$

[117] Li X et al 2019 Terahertz field-induced ferroelectricity in quantum paraelectric $\mathrm{SrTiO}_{3}$ Science 3641079

[118] Nova T F, Disa A S, Fechner M and Cavalleri A 2019 Metastable ferroelectricity in optically strained $\mathrm{SrTiO}_{3} \mathrm{Sci}$ ence 3641075

[119] Zhang S J et al 2020 Photoinduced nonequilibrium response in underdoped $\mathrm{YBa}_{2} \mathrm{Cu}_{3} \mathrm{O}_{6+\mathrm{x}}$ probed by time-resolved terahertz spectroscopy Phys. Rev. X 10 011056

[120] Beaurepaire E, Merle J C, Daunois A and Bigot J Y 1996 Ultrafast spin dynamics in ferromagnetic nickel Phys. Rev. Lett. $764250-3$

[121] Kirilyuk A, Kimel A V and Rasing T 2010 Ultrafast optical manipulation of magnetic order Rev. Mod. Phys. 82 2731-84

[122] Hofherr M et al 2020 Ultrafast optically induced spin transfer in ferromagnetic alloys Sci. Adv. 6 eaay8717

[123] Wilson J A, Di Salvo F J and Mahajan S 1974 Charge-density waves in metallic, layered, transition-metal dichalcogenides Phys. Rev. Lett. 32 882-5

[124] Eichberger M et al 2010 Snapshots of cooperative atomic motions in the optical suppression of charge density waves Nature 468 799-802

[125] Anisimov S I, Kapeliovich B L and Perel'man T L 1974 Electron emission from metal surfaces exposed to ultrashort laser pulses Sov. Phys - FETP 39 375-7

[126] Karl R M et al 2018 Full-field imaging of thermal and acoustic dynamics in an individual nanostructure using tabletop high harmonic beams Sci. Adv. 4 eaau4295

[127] Porter C L et al 2017 General-purpose, wide field-of-view reflection imaging with a tabletop $13 \mathrm{~nm}$ light source Optica 4 1552-7

[128] Zhang B et al 2015 High contrast 3D imaging of surfaces near the wavelength limit using tabletop EUV ptychography Ultramicroscopy 158 98-104

[129] Jacobsen C 2000 X-ray microscopy Proc. 6th Int. Conf. vol 12

[130] Fienup J R 1978 Reconstruction of an object from modulus of its Fourier-transform Opt. Lett. 3 27-9

[131] Miao J W, Charalambous P, Kirz J and Sayre D 1999 Extending the methodology of x-ray crystallography to allow imaging of micrometre-sized non-crystalline specimens Nature $400342-4$

[132] Maiden A M and Rodenburg J M 2009 An improved ptychographical phase retrieval algorithm for diffractive imaging Ultramicroscopy 109 1256-62

[133] Shapiro D A et al 2014 Chemical composition mapping with nanometre resolution by soft x-ray microscopy Nat. Photon. $8765-9$

[134] Takahashi Y et al 2010 High-resolution projection image reconstruction of thick objects by hard x-ray diffraction microscopy Phys. Rev. B 82214102

[135] Xu R et al 2014 Single-shot three-dimensional structure determination of nanocrystals with femtosecond x-ray freeelectron laser pulses Nat. Commun. 54061

[136] Pfeiffer F 2018 X-ray ptychography Nat. Photon. 12 9-17

[137] Lucchini M et al 2015 Ptychographic reconstruction of attosecond pulses Opt. Express 23 29502-13

[138] Frazer T D et al 2019 Engineering nanoscale thermal transport: size- and spacing-dependent cooling of nanostructures Phys. Rev. Appl. 11024042 
[139] Batey D J, Claus D and Rodenburg J M 2014 Information multiplexing in ptychography Ultramicroscopy 138 13-21

[140] Rana A et al 2019 Ptychographic coherent diffractive imaging for attosecond pulses (arXiv:1907.00411)

[141] Lo Y H et al 2019 Multimodal x-ray and electron microscopy of the Allende meteorite $S c i$. Adv. 5 eaax3009
[142] Liao C-T et al 2019 SQUARREL: scattering quotient analysis to retrieve the ratio of elements in x-ray ptychography Microsc. Microanal. 25 112-3

[143] Karl R M et al 2018 Full-field imaging of thermal and acoustic dynamics in an individual nanostructure using tabletop high harmonic beams $S c i$. Adv. 4 eaau4295 